\title{
Non-Ulrich representation type
}

\author{
Daniele Faenzi, Francesco Malaspina and Giangiacomo Sanna
}

\begin{abstract}
We show a remarkable property of the CM-wild variety $\mathbb{P}^{1} \times \mathbb{P}^{2}$, namely that the only ACM sheaves moving in positive-dimensional families are Ulrich bundles. A complete classification of the non-Ulrich range is given.

We prove that this feature is unique in the sense that any other ACM reduced closed subscheme $X \subset \mathbb{P}^{N}$ of dimension $n \geqslant 1$ belongs to the well-known list of CM-finite or CM-tame varieties, or else it remains CM-wild upon removing Ulrich sheaves.
\end{abstract}

\section{Introduction}

Given a reduced closed subscheme $X \subset \mathbb{P}^{N}$ of dimension $n>0$ over an algebraically closed field $\mathrm{k}$, we say that $X$ is arithmetically Cohen-Macaulay (ACM) if its homogeneous coordinate algebra $\mathrm{k}[X]$ is a graded Cohen-Macaulay ring. A coherent sheaf $\mathcal{E}$ on $X$ is ACM if the module $E$ of global sections of $\mathcal{E}$ is a maximal Cohen-Macaulay (MCM) module over $\mathbb{k}[X]$.

A few ACM varieties $X$ support only finitely many isomorphism classes of indecomposable ACM sheaves (up to twist); in this case, $X$ is of finite CM representation type, or CM-finite. These varieties are classified in [EH88] and turn out to be projective spaces, smooth quadrics, rational normal curves, the Veronese surface in $\mathrm{P}^{5}$ and the rational surface scroll of degree 3 in $\mathrm{P}^{4}$. All ACM subvarieties $X$ besides these cases are $C M$-infinite. In a few cases, $X$ supports only discrete families of non-isomorphic indecomposable ACM sheaves. This happens for quadrics of corank 1 with $\operatorname{char}(\mathbb{k}) \neq 2$ (see [BGS87, Section 4]) and $t$-chains of rational curves, that is, $A_{t}$-configurations of smooth rational projective curves, for $t \geqslant 2$ (see [DG01]). We call these varieties CM-discrete (although some authors call these varieties CM-finite as well).

Some CM-non-discrete varieties support at most 1-dimensional families of isomorphism classes of indecomposable ACM sheaves. In dimension 1, this happens when $X$ is an elliptic curve (by [Ati57]) or, in view of [DG01], an $\tilde{A}_{t}$-configuration of smooth rational projective curves for $t \geqslant 2$ or a rational projective curve with a single simple node (which one may think of as an $\tilde{A}_{1}$-configuration). These curves are called cycles of rational curves. In higher dimension, this happens when $X$ is a rational surface scroll of degree 4 in $\mathbb{P}^{5}$; see [FM17]. A variety $X$ with this property is of tame CM-type.

Received 27 January 2020, accepted in final form 20 November 2020.

2020 Mathematics Subject Classification 14J60, 16G60 (primary) 13C14, 14F08, 14J20, 14J45, 16H05, 16G50 (secondary).

Keywords: Cohen-Macaulay modules, arithmetically Cohen-Macaulay sheaves, Ulrich sheaves, moduli of vector bundles, wild representation type.

This journal is (C) Foundation Compositio Mathematica 2021. This article is distributed with Open Access under the terms of the Creative Commons Attribution Non-Commercial License, which permits non-commercial reuse, distribution, and reproduction in any medium, provided that the original work is properly cited. For commercial re-use, please contact the Foundation Compositio Mathematica.

F.M. was partially supported by GNASA-INdAM and MIUR grant Dipartimenti di Eccellenza 2018-2022 (E11G18000350001). D.F. is partially supported by ISITE-BFC project Motivic Invariants of Algebraic Varieties ANR-1S-IDEX-OOOB and EIPHI ANR-17-EURE-0002. 


\section{Faenzi, F. Malaspina and G. Sanna}

As opposed to the previous kinds of varieties, whose CM-categories are under control, one introduces $C M$-wild varieties. In terms of representation theory of algebras, $X$ is of CM-wild type if the category of finitely generated modules over any finitely generated associative $\mathrm{k}$-algebra $\Lambda$ admits a representation embedding into the category of graded MCM modules over $\mathbb{k}[X]$. This means that there is an exact functor $\Phi$ that carries finitely generated $\Lambda$-modules to graded MCM $\mathbb{k}[X]$-modules such that given finitely generated $\Lambda$-modules $M$ and $M^{\prime}$, an isomorphism $\Phi(M) \simeq \Phi\left(M^{\prime}\right)$ implies $M \simeq M^{\prime}$ and $\Phi(M)$ is indecomposable whenever $M$ is. We refer, for example, to [SS07, Chapter XIX] and [DG01] for more precise definitions of the tame and wild representation types of algebras.

The category of ACM sheaves over a CM-wild variety is at least as rich as the category of finitely generated modules of an arbitrary finitely generated associative algebra. It is clear that if $X$ is CM-wild in the algebraic sense, then $X$ supports families of pairwise non-isomorphic indecomposable ACM sheaves of arbitrarily large dimension, so $X$ is CM-wild in the geometric sense.

The main result of [FP15] asserts that all ACM integral closed subschemes in $\mathbb{P}^{N}$ which are not in the list of CM-finite, CM-discrete or CM-tame varieties mentioned above are CM-wild in the algebraic sense.

Among ACM sheaves, a special role is played by Ulrich sheaves. These are characterized by the linearity of the minimal graded free resolution over the polynomial ring of their module of global sections. Ulrich sheaves, originally studied for computing Chow forms, conjecturally exist over any variety (we refer to [ESW03]). They are important for Boij-Söderberg theory (cf. [ES09, SE10]) and for the determination of the representation type of varieties (see [FP15]).

Over many smooth algebraic varieties, heuristics about Ulrich sheaves point out that, among ACM sheaves of a fixed rank, they frequently move in the largest families; that is, the dimension of their deformation space is maximal among such sheaves. For instance, Fano threefolds of Picard number 1 and index at least 2 admit ACM sheaves of rank 2; most of them are semistable, and their moduli space has the largest dimension precisely in the case of Ulrich sheaves (see [BF11]). This also happens on some Fano threefolds of higher Picard rank (we refer, for example, to [CFM18] and references therein). The above considerations motivate the belief that when $X$ is CM-wild, there should exist families of pairwise non-isomorphic indecomposable Ulrich sheaves of arbitrarily large dimension (so $X$ should be Ulrich wild). Algebraically, it should be possible to construct the representation embedding $\Phi$ in such a way that it lands into the category of Ulrich (also known as maximally generated) MCM modules.

The present paper is devoted to further studying the impact of Ulrich sheaves on the representation type of varieties, mostly smooth ones. Namely, taking for granted the slogan that Ulrich sheaves should move in the largest families, we ask what happens if we exclude them: does the representation type of $X$ change? More precisely, can $X$ be downgraded to a CM-finite or CM-tame variety if we require that the image of the representation embedding $\Phi$ contain only finitely many Ulrich modules for any given rank, so that $X$ is algebraically non-Ulrich CM-wild? In particular, are there unbounded families of indecomposable ACM sheaves on $X$ which are not Ulrich?

Our first main contribution is that the answer to this question is negative, except for the two smooth CM-tame surfaces and for a single CM-wild variety, which is $\mathbb{P}^{1} \times \mathbb{P}^{2}$. More specifically, after excluding Ulrich sheaves, the two rational scrolls of degree 4 and $\mathbb{P}^{1} \times \mathbb{P}^{2}$ become of finite CM representation type, while all other varieties keep their representation type unchanged. This holds for all reduced ACM varieties of dimension $n \geqslant 1$ when $\operatorname{char}(\mathbb{k}) \neq 2$. 


\section{NON-UlRICH REPRESENTATION TYPE}

Theorem A. Let $X \subset \mathbb{P}^{N}$ be a reduced closed non-degenerate ACM subscheme of dimension $n \geqslant 1$. Then $X$ is algebraically non-Ulrich CM-wild unless $X$ is

i) a linear space,

ii) a quadric hypersurface of corank at most 1,

iii) an $A_{t}$-configuration of smooth rational curves, for some $t \geqslant 1$,

iv) an $\tilde{A}_{t}$-configuration of smooth rational curves, for $t \geqslant 1$, or an elliptic curve,

v) a surface scroll of degree $d$ in $\mathbb{P}^{d+1}$ with $d \in\{3,4\}$,

vi) the Segre product $\mathbb{P}^{1} \times \mathbb{P}^{2}$ in $\mathbb{P}^{5}$.

In cases v) and vi), the subscheme $X$ supports only finitely many non-Ulrich ACM sheaves.

For the second theorem, we use suitably chosen sets of generators of the derived category of coherent sheaves over projective bundles over $\mathbb{P}^{1}$ to obtain a complete classification of the ACM indecomposable bundles (Ulich or not) over $\mathbb{P}^{1} \times \mathbb{P}^{2}$ and over quartic scrolls. This second case is actually a direct extrapolation from [FM17], so the main point is to treat $\mathbb{P}^{1} \times \mathbb{P}^{2}$, embedded as a degree 3 submanifold of $\mathbb{P}^{5}$ via the Segre product. To state the result, let us introduce some notation. Consider the projection $\pi$ from $X=\mathbb{P}^{1} \times \mathbb{P}^{2}$ to $\mathbb{P}^{1}$, and put $F$ for the divisor class of a fibre of $\pi$ and $L$ for the pull-back of the class of a line on $\mathbb{P}^{2}$. Set $\Omega_{\pi}$ for the cotangent bundle of $\mathbb{P}^{2}$, pulled back to $X$.

Theorem B. Let $\mathcal{F}$ be an indecomposable $A C M$ sheaf on $\mathbb{P}^{1} \times \mathbb{P}^{2}$; assume $\mathrm{H}^{0}(\mathcal{F})=0$ and $\mathrm{H}^{0}(\mathcal{F}(1)) \neq 0$. Then $\mathcal{F}$ is

i) either an Ulrich bundle of the form

$$
0 \rightarrow \mathcal{O}_{X}(-F)^{\oplus a} \rightarrow \mathcal{F} \rightarrow \mathcal{O}_{X}(F-L)^{\oplus b} \rightarrow 0 \quad \text { for some } a, b \in \mathbb{N},
$$

ii) or $\mathcal{O}_{X}(-1)$ or $\mathcal{O}_{X}(-L)$ or the Ulrich bundle $\Omega_{\pi}(L)$.

This has the following surprising corollaries.

Corollary C. Given a polynomial $p \in \mathbb{Q}[t]$, any non-empty moduli space of $H$-semistable ACM sheaves on $X$ with Hilbert polynomial $p$ is a finite set of points.

Put $c_{0}=0, c_{1}=1, c_{k+2}=3 c_{k+1}-c_{k}$ and $c_{-k}=c_{k}$ for all $k \geqslant 0$. The numbers $c_{k}$ are the odd terms of the Fibonacci sequence.

Corollary D. For any $k \in \mathbb{Z}$, there is a unique indecomposable sheaf $\mathfrak{U}_{k}$ fitting into

$$
0 \rightarrow \mathcal{O}_{X}(-F)^{\oplus c_{k-1}} \rightarrow \mathcal{U}_{k} \rightarrow \mathcal{O}_{X}(F-L)^{\oplus c_{k}} \rightarrow 0 .
$$

The sheaves $\mathfrak{U}_{k}$ are Ulrich and rigid and satisfy

$$
\mathcal{U}_{k}^{\vee} \otimes \omega_{X}(2) \simeq \mathcal{U}_{1-k}
$$

Up to a twist by $\mathcal{O}_{X}(t)$, any rigid indecomposable $A C M$ sheaf on $X$ is isomorphic to $\mathcal{O}_{X}$, or to $\mathcal{O}_{X}(-L)$, or to $\Omega_{\pi}(L)$, or to $\mathcal{U}_{k}$ for some $k$.

The paper is organized as follows. We start by recalling some basic notions and preparing the proof of our main results in Section 2, where we quickly sketch how to deal with the case of curves by following the literature. In Section 3, we provide a result ensuring the existence of unbounded families of ACM non-Ulrich sheaves under certain conditions; this is actually a slight modification 


\section{Faenzi, F. Malaspina and G. Sanna}

of [FP15, Theorem A]. Sections 4 and 5 are devoted to the proof of Theorem A in dimension 2 and higher, with the exception of the statement concerning $\mathbb{P}^{1} \times \mathbb{P}^{2}$. More specifically, Section 5 proves it for varieties of minimal degree (except for $\mathbb{P}^{1} \times \mathbb{P}^{2}$ ), that is, non-degenerate integral varieties $X \subset \mathbb{P}^{N}$ of dimension $n \geqslant 2$ and degree $d=N-n+1$, while Section 4 proves it when $d>N-n+1$, the special case $n=2$ being treated in Section 4.1, separately from the range $n \geqslant 3$ showing up in Section 4.2. Finally, in Section 6 we analyze ACM bundles on the exceptional case mentioned above, namely the Segre product $\mathbb{P}^{1} \times \mathbb{P}^{2} \subset \mathbb{P}^{5}$. Theorem B is proved in Section 6.3; cf. in particular Theorem 6.3. The two corollaries above are proved in Section 6.3.1.

\section{Basic facts}

Let $\mathrm{k}$ be a field. Given an integer $N$, set $\mathbb{P}^{N}$ for the projective space of hyperplanes through the origin of $\mathbb{k}^{N+1}$.

\subsection{Notation and conventions}

Let $X \subset \mathbb{P}^{N}$ be a closed integral subscheme of dimension $n$. We assume throughout the paper that $X$ is non-degenerate; namely, there is no hyperplane of $\mathbb{P}^{N}$ that contains $X$. The variety $X$ is equipped with the very ample line bundle $\mathcal{O}_{X}(1)$ defined as the restriction of $\mathcal{O}_{\mathbb{P}^{N}}(1)$ via the embedding $X \subset \mathbb{P}^{N}$. We will write $H$ for the divisor class of $\mathcal{O}_{X}(1)$.

The coordinate ring $R$ of $\mathbb{P}^{N}$ is the graded polynomial algebra in $N+1$ variables with the standard grading, namely $R=\mathbb{k}\left[x_{0}, \ldots, x_{N}\right]$. The homogeneous coordinate ring $\mathbb{k}[X]$ is the graded algebra $\mathbb{k}[X]=R / I_{X}$, where $I_{X}$ is the homogeneous radical ideal of polynomials vanishing on $X$.

The degree of $X$ is computed via the Hilbert polynomial of $I_{X}$. We will be denoted it by $d$.

\subsection{Cohen-Macaulay and Ulrich conditions}

Given a coherent sheaf $\mathcal{E}$ on $X$, the $i$ th cohomology module of $\mathcal{E}$ is the $\mathbb{k}[X]$-module

$$
\mathrm{H}_{*}^{i}(\mathcal{E})=\bigoplus_{k \in \mathbb{Z}} \mathrm{H}^{i}\left(X, \mathcal{E} \otimes \mathcal{O}_{X}(k)\right)
$$

For $i \geqslant 1$, the $\mathbb{k}[X]$-modules $\mathrm{H}_{*}^{i}(\mathcal{E})$ are artinian.

Definition 2.1. A coherent sheaf $\mathcal{E}$ on $X$ is called $A C M$, standing for arithmetically CohenMacaulay, if $\mathcal{E}$ is locally Cohen-Macaulay on $X$ and

$$
\mathrm{H}_{*}^{i}(\mathcal{E})=0 \quad \text { for } i \in\{1, \ldots, n-1\} .
$$

Equivalently, the minimal graded free resolution of the module of global sections $E=\mathrm{H}_{*}^{0}(\mathcal{E})$, seen as an $R$-module, has length $N-n$.

A locally Cohen-Macaulay sheaf on a smooth scheme is locally free; we also call it a vector bundle or simply a bundle.

The variety $X$ itself is said to be ACM if $X$ is projectively normal and $\mathcal{O}_{X}$ is ACM. This is equivalent to asking that $\mathbb{k}[X]$ be a graded Cohen-Macaulay ring, which in turn amounts to the fact that the minimal graded free resolution of $\mathbb{k}[X]$ as $R$-module has length $N-n$. In this case, 


\section{NON-ULRICH REPRESENTATION TYPE}

the line bundles $\mathcal{O}_{X}(k)$ are ACM.

Definition 2.2. Let $d$ be the degree of the embedded variety $X \subset \mathbb{P}^{N}$. For $r>0$, a rank $r$ ACM sheaf $\mathcal{E}$ on $X$ is said to be Ulrich if there is a $t \in \mathbb{Z}$ such that $\mathrm{H}^{0}(X, \mathcal{E}(t-1))=0$ and $h^{0}(X, \mathcal{E}(t))=r d$. We say that $\mathcal{E}$ is initialized by $t$ (we omit "by $t$ " if $t=0$ ).

Given a coherent sheaf $\mathcal{E}$ on $X$, asking that $\mathcal{E}$ be initialized and Ulrich is tantamount to requiring $\mathrm{H}^{*}(X, \mathcal{E}(-j))=0$ for all $1 \leqslant j \leqslant n$; cf. [ES09, Proposition 2.1].

Remark 2.3. We should warn the reader that the usual definition of Ulrich sheaf in the literature is equivalent to our definition of initialized Ulrich sheaf. We adopted this slightly different definition in order to work with sheaves which are Ulrich up to a twist.

\subsection{Semistability}

Let $X \subset \mathbb{P}^{N}$ be a closed subscheme of dimension $n>0$ embedded by the very ample divisor $H$. Stability of sheaves on $X$ will always mean Gieseker stability of pure $n$-dimensional sheaves with respect to the divisor $H$.

The Hilbert polynomial of a coherent sheaf $\mathcal{E}$ on $X$, computed with respect to $H$, is denoted by $P(\mathcal{E}, t)$. The rank of $\mathcal{E}$ is defined as the element $r \in \mathbb{Q}$ such that the leading coefficient of $P(\mathcal{E}, t)$ equals $r d / n$ !. For $r \neq 0$, we write $\mathrm{p}(\mathcal{E}, t):=P(\mathcal{E}, t) / r$ for the reduced Hilbert polynomial of $\mathcal{E}$.

Given polynomials $p, q \in \mathbb{Q}[t]$, we write $p \preceq q$ if $p(t) \leqslant q(t)$ for $t \gg 0$. A coherent sheaf $\mathcal{E}$ of rank $r \neq 0$ is semistable if it is pure (that is, all its subsheaves have support of dimension $n$ ) and for any non-zero subsheaf $\mathcal{F} \subsetneq \mathcal{E}$, we have $\mathrm{p}(\mathcal{F}, t) \preceq \mathrm{p}(\mathcal{E}, t)$. Stability is defined by strict inequalities.

A coherent sheaf $\mathcal{E}$ on $X$ is simple if $\operatorname{Hom}_{X}(\mathcal{E}, \mathcal{E})$ is generated by $\mathrm{id}_{\mathcal{E}}$.

\subsection{Basic remarks on Theorem A}

Here are some comments about Theorem A from the introduction. Again, we assume that $X \subset \mathbb{P}^{N}$ is an $n$-dimensional closed subscheme over a field $\mathrm{k}$, with $n \geqslant 1$.

Remark 2.4. To obtain the CM-wildness of $X$, it suffices to find a representation embedding of some algebra of wild representation type into the category of ACM sheaves on $X$ (cf. for instance [FP15, Section 1.2]). We will mostly take such an algebra to be the free k-algebra in two generators or the path algebra of the Kronecker quiver with two vertices and three arrows.

Remark 2.5. In the setting of Theorem A (that is, $X \subset \mathbb{P}^{N}$ is reduced, closed, non-degenerate, $\mathrm{ACM}$ and $\mathrm{k}$ is algebraically closed), the restriction $\operatorname{char}(\mathbb{k}) \neq 2$ is only needed to deal with the case of quadric hypersurfaces of corank 1, which is derived from [BGS87], so the result is also valid in characteristic 2 except perhaps for this case. More information on MCM modules on quadrics in characteristic 2 can be found in [BEH87].

Remark 2.6. The statement for curves in Theorem A is a consequence of [DG01]; cf. also [BDG01, BBDG06]. It should be pointed out that the cohomological vanishing required for a sheaf to be ACM plays no role in dimension 1 , so the statement is really about locally Cohen-Macaulay sheaves. 


\section{Faenzi, F. Malaspina and G. Sanna}

More in detail, given a reduced connected projective curve $X \subset \mathbb{P}^{N}$ of degree $d$ over an algebraically closed field $\mathrm{k}$, in order to check that $X$ is non-Ulrich CM-wild, it suffices to find a representation embedding of some wild k-algebra into the category of vector bundles over $X$ in such a way that for any given rank $r$, the resulting rank $r$ bundles $\mathcal{E}$ satisfy

$$
\mathrm{H}^{0}(\mathcal{E}(-H))=0, \quad 0 \neq \operatorname{dim} \mathrm{H}^{0}(\varepsilon) \neq d r
$$

except for finitely many choices of $\mathcal{E}$.

In turn, this is already the case for all vector bundles appearing in [BBDG06, Theorem 7] if $X$ has arithmetic genus $g>1$. Indeed, such bundles have degree $r$, so (2.1) follows easily from the Riemann-Roch theorem.

For curves of arithmetic genus $g \leqslant 1$ which are not of type $A_{t}$ or $\tilde{A}_{t}$, the construction of [DG01] provides bundles $\mathcal{E}$ whose pull-backs under the normalization $\pi: \tilde{X} \rightarrow X$ decompose as a direct sum $\oplus_{i=1}^{t} \varepsilon_{i}$, where $\left(\tilde{X}_{i} \mid i=1, \ldots, t\right)$ is the set of irreducible components of $\tilde{X}$ and, for each $i \in\{1, \ldots, t\}$, the sheaf $\mathcal{E}_{i}$ is a vector bundle on $\tilde{X}_{i}$. One checks that the degrees of the bundles $\left(\mathcal{E}_{i} \mid i=1, \ldots, t\right)$ can be chosen in such a way that $\pi^{*}(\mathcal{E})$ and hence $\mathcal{E}$ satisfy (2.1) by the Riemann-Roch theorem.

In the same way, the freedom in the choice of $\operatorname{deg}\left(\mathcal{E}_{i}\right)_{i}$ allows us to define infinitely indecomposable vector bundles which are not isomorphic up to a twist over $A_{t}$-configurations for $t \geqslant 2$, as well as 1-parameter family thereof over elliptic curves and $\tilde{A}_{t}$-configurations for $t \geqslant 1$.

In view of the previous remark, in the proof of Theorem A, we will be allowed to assume that the dimension $n$ of $X$ is at least 2 .

\section{Representation embeddings and non-Ulrich sheaves}

Let $X \subset \mathbb{P}^{N}$ be a non-degenerate closed subscheme of dimension $n>0$ over a field $\mathbb{k}$. We propose here a criterion, based on classical ideas about extensions of sheaves and modules, for $X$ to be non-Ulrich CM-wild. Since this does not really depend on $X$ being smooth or ACM, we formulate it in a more general setting than what is actually needed to prove Theorem A. The result is a slight modification of [FP15, Theorem A].

Theorem 3.1. Let $\mathcal{A}$ and $\mathcal{B}$ be simple semistable $A C M$ sheaves such that $\mathrm{p}(\mathcal{B}) \prec \mathrm{p}(\mathcal{A})$, and assume $\operatorname{dim}_{\mathbb{k}} \operatorname{Ext}_{X}^{1}(\mathcal{B}, \mathcal{A}) \geqslant 3$. Then the following hold:

i) The subscheme $X$ is $C M$-wild.

ii) If $n \geqslant 2$ and $\mathcal{A}$ and $\mathcal{B}$ are not Ulrich initialized by the same integer, then $X$ is algebraically non-Ulrich CM-wild.

iii) The same conclusion as in part ii) also holds for $n=1$ if there is no $t \in \mathbb{Z}$ such that $\mathrm{H}^{0}(X, \mathcal{A}(t))=\mathrm{H}^{1}(X, \mathcal{B}(t))=0$.

Proof. We use the setting and notation of [FP15, Theorem A]. To be in position to apply that result, we should verify that any non-zero morphism $\mathcal{A} \rightarrow \mathcal{B}$ is an isomorphism. But this is obvious since $\mathrm{p}(\mathcal{B}) \prec \mathrm{p}(\mathcal{A})$ and $\mathcal{A}$ and $\mathcal{B}$ are semistable, so any morphism $\mathcal{A} \rightarrow \mathcal{B}$ is actually zero, so part i) is clear.

Therefore, $X$ is algebraically CM-wild. Now, assume that no integer $t$ turns $\mathcal{A}(t)$ and $\mathcal{B}(t)$ into initialized Ulrich sheaves. Recall that, by construction, the sheaves appearing in the families provided by [FP15, Theorem A] are extensions of copies of $\mathcal{A}$ and $\mathcal{B}$. If a sheaf $\mathcal{E}$ is an extension 


\section{NON-ULRICH REPRESENTATION TYPE}

of say $a$ copies of $\mathcal{A}$ and $b$ copies of $\mathcal{B}$, it suffices to prove that $\mathcal{E}$ is actually non-Ulrich when $a$ and $b$ are both non-zero.

To check this, in order to obtain a contradiction, we let $t$ be an integer that initializes $\mathcal{E}$ as an Ulrich sheaf, that is, such that $\mathrm{H}^{*}(X, \mathcal{E}(t-j))=0$ for all $1 \leqslant j \leqslant n$. Since $\mathcal{A}$ and $\mathcal{B}$ are ACM by assumption, we have the vanishing $\mathrm{H}^{i}(X, \mathcal{A}(t-j))=\mathrm{H}^{i}(X, \mathcal{B}(t-j))$ for $1 \leqslant i \leqslant n-1$ and for all $j \in \mathbb{Z}$.

By definition, $\mathcal{E}$ fits into an exact sequence of the form

$$
0 \rightarrow \mathcal{A}^{\oplus a} \rightarrow \mathcal{E} \rightarrow \mathcal{B}^{\oplus b} \rightarrow 0
$$

where we may assume $a \neq 0 \neq b$. Therefore, from the vanishing $\mathrm{H}^{i}(X, \mathcal{E}(t-j))=0$, we deduce $\mathrm{H}^{0}(X, \mathcal{A}(t-j))=0=\mathrm{H}^{n}(X, \mathcal{B}(t-j))$ for $1 \leqslant j \leqslant n$.

Now, if $n \geqslant 2$, then because $\mathcal{A}$ is $\mathrm{ACM}$, the vanishing $\left.\mathrm{H}^{1}(X, \mathcal{A}(t-j))\right)$ takes place for all $j \in \mathbb{Z}$, so we see that $\mathrm{H}^{0}(X, \mathcal{E}(t-j))=0$ implies $\mathrm{H}^{0}(X, \mathcal{B}(t-j))=0$ for $1 \leqslant j \leqslant n$. This implies that $\mathcal{B}$ is Ulrich, initialized by $t$, and similarly we get that this holds true for $\mathcal{A}$. But this is excluded, and we conclude that part ii) holds.

With the same set-up, we can also prove part iii). Indeed, when $X$ is a curve, a coherent sheaf $\mathcal{E}$ is Ulrich if and only if there is a $t \in \mathbb{Z}$ such that $\mathrm{H}^{i}(X, \mathcal{E}(t))=0$ for all $i$, which implies $\mathrm{H}^{0}(X, \mathcal{A}(t))=0$ and $\mathrm{H}^{1}(X, \mathcal{B}(t))=0$. But our assumption implies that there is no $t \in \mathbb{Z}$ such that $\mathrm{H}^{0}(X, \mathcal{A}(t))$ and $\mathrm{H}^{1}(X, \mathcal{B}(t))$ vanish together, so $\mathcal{E}$ is not Ulrich.

\section{Varieties of non-minimal degree}

Let $X \subset \mathbb{P}^{N}$ be a non-degenerate closed subscheme of dimension $n$ over an algebraically closed field $\mathbb{k}$. Assume that $X$ is reduced and ACM. Put $d=\operatorname{deg}(X)$.

We mentioned in Section 2.4 that in order to prove Theorem A, we can assume $n \geqslant 2$. In this section, we would like to treat the case when $X$ is not of minimal degree, which is to say $d \geqslant N-n+2$.

We first look at the case $(n, d)=(2, N)$, so $X$ is a surface of quasi-minimal degree, which we deal with in the next paragraph. The remaining cases are basically already in [FP15], up to the result, proved in Section 4.2, that the $c$ th syzygy of an ACM sheaf supported on a $c$-codimensional linear section is an ACM sheaf which is never Ulrich when $d \geqslant N-n+2$.

\subsection{Surfaces of quasi-minimal degree}

For this subsection, $X \subset \mathbb{P}^{N}$ is an ACM reduced closed surface of degree $N$, so $X$ is of quasiminimal degree. It turns out that $X$ is locally Gorenstein, namely $\omega_{X} \simeq \mathcal{O}_{X}(-1)$; see [Mig98, Corollary 4.1.5].

Let us first observe that if $X$ is reducible, then $X$ is non-Ulrich CM-wild. Indeed, in view of [FP15, Section 7.1] and since $n \geqslant 2$, we are in position to apply [FP15, Theorem 5.2] and show that $X$ is CM-wild. However, the sheaves obtained in this way are not Ulrich. Indeed, such a sheaf $\mathcal{E}$ appears as an extension of an ACM sheaf $\mathcal{F}_{1}(q)$ on a first component $X_{1}$ of $X$ for some $q>0$, and of the structure sheaf of a second component $X_{2}$ of $X$, and the resulting sheaf $\mathcal{E}$ is not Ulrich for $q \gg 0$. So we may assume until the end of the subsection that $X$ is integral. 


\section{Faenzi, F. Malaspina and G. Sanna}

4.1.1 Syzygies of Ulrich bundles. Let $X$ be an integral ACM surface of quasi-minimal degree. We first assume that $X$ is not a cone. In view of [FP15, Section 7.4], there exist stable initialized Ulrich bundles $\mathcal{E}_{1}$ and $\mathcal{E}_{2}$ of rank 2 on $X$ and determinant $\mathcal{O}_{X}(2)$ such that

$$
\begin{aligned}
& \operatorname{Hom}_{X}\left(\mathcal{E}_{i}, \mathcal{E}_{i}\right)=\operatorname{kid}_{\varepsilon_{i}} \quad \text { for } i \in\{1,2\}, \\
& \operatorname{Hom}_{X}\left(\varepsilon_{i}, \mathcal{E}_{j}\right)=0 \quad \text { if }\{i, j\}=\{1,2\} \text {, } \\
& \operatorname{dim} \operatorname{Ext}_{X}^{1}\left(\mathcal{E}_{i}, \mathcal{E}_{i}\right)=5 \quad \text { for } i \in\{1,2\} \text {, } \\
& \operatorname{dim} \operatorname{Ext}_{X}^{1}\left(\mathcal{E}_{i}, \mathcal{E}_{j}\right)=4 \text { if }\{i, j\}=\{1,2\} \text {, } \\
& \operatorname{Ext}_{X}^{p}\left(\mathcal{E}_{i}, \mathcal{E}_{j}\right)=0 \quad \text { for } i, j \in\{1,2\} \text { and } p \geqslant 2 \text {. }
\end{aligned}
$$

Consider the evaluations of global sections for $i \in\{1,2\}$,

$$
e_{\varepsilon_{i}}: H^{0}\left(\mathcal{E}_{i}\right) \otimes \mathcal{O}_{X} \rightarrow \mathcal{E}_{i} .
$$

We define $\mathcal{A}_{i}=\operatorname{ker}\left(e_{\varepsilon_{i}}\right)^{\vee}$ for $i \in\{1,2\}$.

Proposition 4.1. The sheaves $\mathcal{A}_{1}$ and $\mathcal{A}_{2}$ are simple, $A C M$ vector bundles which are not Ulrich. They satisfy

$$
\begin{array}{ll}
\operatorname{Hom}_{X}\left(\mathcal{A}_{i}, \mathcal{A}_{i}\right)=\operatorname{lkid}_{\mathcal{A}_{i}} & \text { for } i \in\{1,2\}, \\
\operatorname{Hom}_{X}\left(\mathcal{A}_{i}, \mathcal{A}_{j}\right)=0 & \text { if }\{i, j\}=\{1,2\}, \\
\operatorname{dim} \operatorname{Ext}_{X}^{1}\left(\mathcal{A}_{i}, \mathcal{A}_{i}\right)=5 & \text { for } i \in\{1,2\}, \\
\operatorname{dim} \operatorname{Ext}_{X}^{1}\left(\mathcal{A}_{i}, \mathcal{A}_{j}\right)=4 & \text { if }\{i, j\}=\{1,2\}, \\
\operatorname{Ext}_{X}^{p}\left(\mathcal{A}_{i}, \mathcal{A}_{j}\right)=0 & \text { for } i, j \in\{1,2\} \text { and } p \geqslant 2 .
\end{array}
$$

Proof. It is well known that the module of global sections of an initialized Ulrich bundle of rank $r$ over $X$ is generated by $N r$ elements of degree 0 . Therefore, the evaluation maps $e_{\varepsilon_{k}}$ are surjective for $k \in\{1,2\}$, and the sheaves $\operatorname{ker}\left(e_{\varepsilon_{k}}\right)$ are locally free and ACM of rank $2(N-1)$ on $X$. In other words, $\mathrm{H}_{*}^{1}\left(\mathcal{A}_{k}^{\vee}\right)=0$ for $k \in\{1,2\}$. Also, by the definition of $e_{\mathcal{E}_{k}}$, we have $\mathrm{H}^{0}\left(\mathcal{A}_{k}^{\vee}\right)=0$ for $k \in\{1,2\}$.

Using the isomorphism $\omega_{X} \simeq \mathcal{O}_{X}(-1)$ and Serre duality, we get that the $\mathcal{A}_{k}$ are ACM bundles on $X$ for $k \in\{1,2\}$. Also, $\mathcal{E}_{k}^{\vee} \simeq \mathcal{E}_{k}(-2)$, so we have

$$
0 \rightarrow \mathcal{E}_{k}(-2) \rightarrow \mathcal{O}_{X}^{\oplus 2 N} \rightarrow \mathcal{A}_{k} \rightarrow 0 .
$$

This gives at once $\mathrm{H}^{0}\left(\mathcal{A}_{k}(-1)\right)=0$ and $\operatorname{dim}\left(\mathrm{H}^{0}\left(\mathcal{A}_{k}\right)\right)=2 N<2 N(N-1)$; hence $\mathcal{A}_{k}$ is not Ulrich for $k \in\{1,2\}$.

For $i, j, k \in\{1,2\}$, since $\mathcal{E}_{j}$ is an initialized Ulrich bundle, we have $\mathrm{H}^{*}\left(\mathcal{E}_{j}(-2)\right)=0$; thus, applying $\operatorname{Hom}_{X}\left(-, \mathcal{E}_{j}(-2)\right)$ to $(4.3)$, we get $\operatorname{Ext}_{X}^{p}\left(\mathcal{E}_{k}, \mathcal{E}_{j}\right) \simeq \operatorname{Ext}_{X}^{p+1}\left(\mathcal{A}_{k}, \mathcal{E}_{j}(-2)\right)$ for all $p \geqslant 0$. Using Serre duality and the vanishing we already proved for $\mathcal{A}_{i}^{\vee}$, we get $\operatorname{Ext}_{X}^{*}\left(\mathcal{A}_{i}, \mathcal{O}_{X}\right)=0$. Therefore, applying $\operatorname{Hom}_{X}\left(\mathcal{A}_{i},-\right)$ to $(4.3)$, we get $\operatorname{Ext}_{X}^{p+1}\left(\mathcal{A}_{i}, \mathcal{E}_{k}(-2)\right) \simeq \operatorname{Ext}_{X}^{p}\left(\mathcal{A}_{i}, \mathcal{A}_{k}\right)$ for all $p \geqslant 0$. Summing up, we get

$$
\operatorname{Ext}_{X}^{p}\left(\mathcal{E}_{i}, \mathcal{E}_{j}\right) \simeq \operatorname{Ext}_{X}^{p}\left(\mathcal{A}_{i}, \mathcal{A}_{j}\right) \text { for all } p \text { and all } i, j \in\{1,2\} .
$$

Hence, (4.2) follows from (4.1), and the proposition is proved.

If $X$ is a cone, then we use the construction of [FP15, Section 7.3]. This gives two initialized 


\section{NON-ULRICH REPRESENTATION TYPE}

Ulrich sheaves $\mathcal{E}_{1}$ and $\mathcal{E}_{2}$ of rank 1 on $X$ such that

$$
\begin{array}{ll}
\operatorname{Hom}_{X}\left(\mathcal{E}_{i}^{\vee}, \mathcal{E}_{i}^{\vee}\right)=\operatorname{lkid}_{\mathcal{E}_{i}} & \text { for } i \in\{1,2\}, \\
\operatorname{Hom}_{X}\left(\mathcal{E}_{i}^{\vee}, \mathcal{E}_{j}^{\vee}\right)=0 & \text { if }\{i, j\}=\{1,2\}, \\
\operatorname{dim} \operatorname{Ext}_{X}^{1}\left(\mathcal{E}_{i}^{\vee}, \mathcal{E}_{i}^{\vee}\right)=N+1 & \text { for } i \in\{1,2\}, \\
\operatorname{dim} \operatorname{Ext}_{X}^{1}\left(\mathcal{E}_{i}^{\vee}, \mathcal{E}_{j}^{\vee}\right)=N & \text { if }\{i, j\}=\{1,2\} .
\end{array}
$$

Since $\mathcal{E} x t_{X}^{1}\left(\mathcal{E}_{i}, \mathcal{O}_{X}\right)=0$ for $i \in\{1,2\}$, this allows us to define two reflexive ACM sheaves $\mathcal{A}_{1}$ and $\mathcal{A}_{2}$ of rank $(N-1)$ as in Proposition 4.1 and show that $\mathcal{A}_{1}$ and $\mathcal{A}_{2}$ are not Ulrich as $\mathrm{H}^{0}\left(\mathcal{A}_{k}(-1)\right)=0$ and $\operatorname{dim}\left(\mathrm{H}^{0}\left(\mathcal{A}_{k}\right)\right)=N<N(N-1)$. Note that for $i \in\{1,2\}$, by Serre duality, we have $\mathrm{H}^{*}\left(\mathcal{E}_{i}^{\vee}\right)=0$ since $\omega_{X} \simeq \mathcal{O}_{X}(-1)$ and $\mathcal{E}_{i}$ is initialized Ulrich. Again, $\operatorname{Ext}_{X}^{p}\left(\mathcal{A}_{i}, \mathcal{O}_{X}\right)=0$ for all $p$ and $i \in\{1,2\}$ so, by the same argument as before, we get

$$
\begin{array}{ll}
\operatorname{Hom}_{X}\left(\mathcal{A}_{i}, \mathcal{A}_{i}\right)=\operatorname{kkid}_{\mathcal{A}_{i}} & \text { for } i \in\{1,2\}, \\
\operatorname{Hom}_{X}\left(\mathcal{A}_{i}, \mathcal{A}_{j}\right)=0 & \text { if }\{i, j\}=\{1,2\}, \\
\operatorname{dim} \operatorname{Ext}_{X}^{1}\left(\mathcal{A}_{i}, \mathcal{A}_{i}\right)=N+1 & \text { for } i \in\{1,2\}, \\
\operatorname{dim} \operatorname{Ext}_{X}^{1}\left(\mathcal{A}_{i}, \mathcal{A}_{j}\right)=N & \text { if }\{i, j\}=\{1,2\} .
\end{array}
$$

Summing up, independently of whether $X$ is a cone or not, in view of [FP15, Theorem A], we get that $X$ is non-Ulrich CM-wild and even strictly CM-wild.

4.1.2 A second construction for del Pezzo surfaces. Let us give a second construction with the further assumption that $X$ is smooth, so $X$ is an anticanonically embedded del Pezzo surface. This construction has the advantage of being self-contained and the drawback of relying on the explicit description of $X$ as a blown-up plane or a quadric surface. More precisely, recall that $X$ is either a blow-up of $\mathbb{P}^{2}$ at $9-d$ points or the product variety $\mathbb{P}^{1} \times \mathbb{P}^{1}$. We construct $\mathrm{ACM}$ bundles (Ulrich or not) on $X$ with the same methods in both cases, only with a slightly different choice of the invariants.

If $X$ is a blow-up of $\mathbb{P}^{2}$, we fix a birational surjective morphism $\pi: X \rightarrow \mathbb{P}^{2}$ and let $\mathcal{O}_{X}(L)=$ $\pi^{*}\left(\mathcal{O}_{\mathbb{P}^{2}}(1)\right)$ and $M=2 L$. Given $(a, b) \in \mathbb{N}^{2}$ with $a \geqslant 2$, we put $D(a, b)=3 a b-a^{2}-b^{2}+1$ and $b_{a}=2 a$. In the second case, we set $\pi_{1}$ and $\pi_{2}$ to be the projection maps onto the two $\mathbb{P}^{1}$-factors and let $\mathcal{O}_{X}(L)=\pi_{1}^{*}\left(\mathcal{O}_{\mathbb{P}^{1}}(1)\right)$ and $\mathcal{O}_{X}(F)=\pi_{2}^{*}\left(\mathcal{O}_{\mathbb{P}^{1}}(1)\right)$. This time, we take $(a, b) \in \mathbb{N}^{2}$ with $a \geqslant 1$, and we put $D(a, b)=4 a b-a^{2}-b^{2}+1, b_{a}=3 a, M=2 L+F$.

Proposition 4.2. Choose $(a, b)$ such that $D(a, b)>0$ and $b \geqslant b_{a}$. Then, for $f$ general enough in $\operatorname{Hom}_{X}\left(\mathcal{O}_{X}(L)^{\oplus b}, \mathcal{O}_{X}(M)^{\oplus a}\right)$, the sheaf $\mathcal{E}=\operatorname{ker}(f)$ is simple, locally free and ACM, with $\operatorname{dim} \operatorname{Ext}_{X}^{1}(\mathcal{E}, \mathcal{E})=D(a, b)$ and $\operatorname{Ext}_{X}^{2}(\mathcal{E}, \mathcal{E})=0$; the sheaf $\mathcal{E}$ is not Ulrich when $b>b_{a}$.

Proof. Note that $b \geqslant b_{a} \geqslant a$ and that the locally free sheaf $\mathcal{H}=\mathcal{H} m_{X}\left(\mathcal{O}_{X}(L)^{\oplus b}, \mathcal{O}_{X}(M)^{\oplus a}\right) \simeq$ $\mathcal{O}_{X}(M-L)^{\oplus b a}$ is globally generated. Therefore, for a general choice of $f \in \mathrm{H}^{0}(\mathcal{H})$ and for any integer $k \in\{0, \ldots, a-1\}$, the degeneracy locus $D_{k}(f)$ defined by the $(k+1)$-minors of the associated map $f: \mathcal{O}_{X}(L)^{\oplus b} \rightarrow \mathcal{O}_{X}(M)^{\oplus a}$ has codimension $(b-k)(a-k)$ in $X$ in view of a Bertini-type result; see, for instance, [Ott95, Teorema 2.8] or [CCJ21, Lemma 11.6]. In particular, for $k=a-1$, since $b-a+1 \geqslant b_{a}-a+1 \geqslant 2 a-a+1 \geqslant a+1 \geqslant 3$ and $\operatorname{dim}(X)=2$, we have $D_{a-1}(f)=\emptyset$, so $f$ is surjective.

Then, the sheaf $\mathcal{E}=\operatorname{ker}(f)$ is locally free of rank $b-a \geqslant 2$. We write down the exact sequence

$$
0 \rightarrow \mathcal{E} \rightarrow \mathcal{O}_{X}(L)^{\oplus b} \rightarrow \mathcal{O}_{X}(M)^{\oplus a} \rightarrow 0 .
$$




\section{Faenzi, F. Malaspina and G. Sanna}

Next, observe that the $\mathrm{k}$-vector space $\operatorname{Hom}_{X}\left(\mathcal{O}_{X}(L), \mathcal{O}_{X}(M)\right)$ has dimension 3 or 4 depending on whether $X$ is a blow-up of $\mathbb{P}^{2}$ or $X \simeq \mathbb{P}^{1} \times \mathbb{P}^{1}$. In both cases, the assumption $D(a, b)>0$ ensures that [Kac80, Theorem 4] applies (cf. the argument of [CMP12, Proposition 3.5(i)]) and shows that $\mathcal{E}$ is simple if $f$ is general enough. The same argument proves $\operatorname{dim}_{\operatorname{Ext}_{X}^{1}}^{1}(\mathcal{E}, \mathcal{E})=D(a, b)$ and $\operatorname{Ext}_{X}^{2}(\mathcal{E}, \mathcal{E})=0$.

Next, we show that $\mathcal{E}$ is ACM. Note that $\mathcal{O}_{X}(L)$ is ACM for the polarization $H$ and that $\mathrm{H}^{0}\left(\mathcal{O}_{X}(M+t H)\right)=0$ for any integer $t \leqslant-1$, so $(4.4)$ gives $\mathrm{H}^{1}(\mathcal{E}(t H))=0$ for all $t \leqslant-1$. Also, Serre duality gives $\mathrm{H}^{k}\left(\mathcal{O}_{X}(L-H)\right)=\mathrm{H}^{k}\left(\mathcal{O}_{X}(M-H)\right)=0$ for all $k$. So once we make sure that $\mathrm{H}^{1}(\mathcal{E})=0$, we will get that $\mathcal{E}(H)$ is $H$-regular and hence $\mathrm{H}^{1}(\mathcal{E}(t H))=0$ for $t \geqslant 0$, so that $\mathcal{E}$ will be proved to be ACM.

So let us prove that $\mathrm{H}^{1}(\mathcal{E})=0$. If $X$ is a blow-up of $\mathrm{P}^{2}$, this follows from [EH92, Propositions 1.1 and 4.1] in view of the assumption $b \geqslant b_{a}$. When $X \simeq \mathbb{P}^{1} \times \mathbb{P}^{1}$, we first note that the condition $\mathrm{H}^{1}(\mathcal{E})=0$ is open on flat families and that

$$
\left\{\operatorname{ker}(f) \mid f \in \mathrm{H}^{0}(\mathcal{H}) \text { gives a surjective map } \mathcal{O}_{X}(L)^{\oplus b} \rightarrow \mathcal{O}_{X}(M)^{\oplus a}\right\}
$$

defines a family of vector bundles on $X$ which is indeed flat.

In view of this discussion, in order to get the statement for general $f \in \mathrm{H}^{0}(\mathcal{H})$, it suffices to prove it for one choice of $f_{0} \in \mathrm{H}^{0}(\mathcal{H})$, provided that the associated $f_{0}: \mathcal{O}_{X}(L)^{\oplus b} \rightarrow \mathcal{O}_{X}(M)^{\oplus a}$ is surjective. To choose a convenient element $f_{0}$, note that again a Bertini-type argument ensures that for a general choice of $g \in \operatorname{Hom}_{X}\left(\mathcal{O}_{X}(L)^{\oplus 3}, \mathcal{O}_{X}(M)\right)$, the map $g$ is surjective. Define $\mathcal{F}=\operatorname{ker}(g)$, so

$$
0 \rightarrow \mathcal{F} \rightarrow \mathcal{O}_{X}(L)^{\oplus 3} \rightarrow \mathcal{O}_{X}(M) \rightarrow 0 .
$$

Then, choose $f_{0}$ to be a diagonal map consisting of $a$ copies of $g$ as above, completed by $b-b_{a}=$ $b-3 a$ zeroes. We thus get a surjective map $f_{0}: \mathcal{O}_{X}(L)^{\oplus b} \rightarrow \mathcal{O}_{X}(M)^{\oplus a}$, and $\mathcal{E}_{0}=\operatorname{ker}\left(f_{0}\right) \simeq$ $\mathcal{O}_{X}(L)^{b-3 a} \oplus \mathcal{F}^{\oplus a}$.

We still have to prove $\mathrm{H}^{1}\left(\mathcal{E}_{0}\right)=0$. To do it, it suffices to show $\mathrm{H}^{1}(\mathcal{F})=0$. In turn, we use an argument analogous to [ESW03, Proposition 5.9] to show this and actually prove that $\mathcal{F}$ is Ulrich on $(X, H)$, where $H=2 L+2 F$. Indeed, $c_{1}(\mathcal{F})=L-F$, so $\mathcal{F} \simeq \mathcal{F}^{\vee}(L-F)$, and the dual of (4.5) yields the exact sequence

$$
0 \rightarrow \mathcal{O}_{X}(-L-2 F) \rightarrow \mathcal{O}_{X}(-F)^{\oplus 3} \rightarrow \mathcal{F} \rightarrow 0 .
$$

This immediately implies $H^{*}(\mathcal{F})=0$. Also, (4.5) gives

$$
0 \rightarrow \mathcal{F}(-H) \rightarrow \mathcal{O}_{X}(-L-2 F)^{\oplus 3} \rightarrow \mathcal{O}_{X}(-F) \rightarrow 0,
$$

which implies $\mathrm{H}^{*}(\mathcal{F}(-H))=0$, so that $\mathcal{F}$ is Ulrich.

We have thus proved that $\mathcal{E}$ is ACM. Finally, $\mathcal{E}$ is not Ulrich when $b>b_{a}$. Indeed, from (4.4), we have $\mathrm{H}^{0}(\mathcal{E}(-H))=0$, so we know that $\mathcal{E}$ is not Ulrich as soon as we show

$$
0<\chi(\mathcal{E})<d(b-a) .
$$

Now, on one hand, the assumption $b>b_{a}$ guarantees $\chi(\mathcal{E})>0$. On the other hand, when $X$ is a blow-up of $\mathbb{P}^{2}$, we get $\chi(\mathcal{E})=3(b-2 a)<3(b-a) \leqslant d(b-a)$, while for $X \simeq \mathbb{P}^{1} \times \mathbb{P}^{1}$ (hence $d=8)$, we have $\chi(\varepsilon)=2(b-4 a)<2(b-a) \leqslant 8(b-a)$. In both cases, the desired equality holds and the statement is proved.

Remark 4.3. The previous proof actually implies that for $X \simeq \mathbb{P}^{1} \times \mathbb{P}^{1}$ embedded by $2 L+2 F$ and $b=3 a$, the sheaf $\mathcal{E}$ obtained by $f$ as in Proposition 4.2 is a simple Ulrich bundle of rank $2 a$. 


\section{NON-ULRICH REPRESENTATION TYPE}

Proposition 4.2 shows the geometric version of Theorem A when $X$ is a del Pezzo surface. Indeed, choosing for instance $b=b_{a}+1$, the above construction provides families of non-Ulrich pairwise non-isomorphic indecomposable bundles, whose dimension grows as a quadratic function of $a$.

\subsection{The higher range}

According to Section 4.1, we have to justify that Theorem A holds for all non-degenerate reduced closed ACM subschemes $X \subset \mathbb{P}^{N}$ of dimension $n \geqslant 2$ and degree $d$ provided that $(d, n, N)$ lie in the higher range, namely $d \geqslant N-n+3$, or $n \geqslant 3$ and $d=N-n+2$. We essentially extract this from [FP15] up to the result, proved below, that syzygies of Ulrich sheaves are never Ulrich.

Indeed, [FP15, Theorem 4.2] already asserts that when a subscheme $X$ of dimension at least 2 as above is in the range $d \geqslant N-n+2$ (that is, $X$ is not of minimal degree), then it is of wild CM-type.

What we do here is to show that the proof of [FP15, Theorem 4.2] already yields non-Ulrich sheaves. Looking into this proof, we see that it proceeds by reduction to a transverse linear section $Y$ of $X$ of dimension 1 in case $d \geqslant N-n+3$, or of dimension 2 in case $d=N-n+2$. Namely, setting $c$ for the codimension of $Y$ in $X$, one first constructs families of arbitrarily large dimension of indecomposable pairwise non-isomorphic ACM sheaves $\mathcal{F}$ on $Y$. Then, one considers a minimal graded free resolution $F$. $\rightarrow F$ of the finitely generated $\mathbb{k}[X]$-module $F=\mathrm{H}_{*}^{0}(\mathcal{F})$, of the form

$$
0 \leftarrow F \leftarrow F_{0} \leftarrow F_{1} \leftarrow \cdots \leftarrow F_{\ell-1} \stackrel{d_{\ell}}{\longleftarrow} F_{\ell} \leftarrow \cdots,
$$

where, for all $i \geqslant 0$, the term $F_{i}$ is a finitely generated free $\mathbb{k}[X]$-module. For $i \geqslant 0$, we write $\Sigma_{i}^{X}(F)$ for $\operatorname{Im}\left(d_{i}\right)$ and $\Sigma_{i}^{X}(\mathcal{F})$ for the sheafification of $\Sigma_{i}^{X}(F)$.

The sheaf $\mathcal{E}=\Sigma_{c}^{X}(\mathcal{F})$ is ACM over $X$. One shows via [FP15, Theorem B] that the families of sheaves $\mathcal{E}$ constructed in this way are still made up of indecomposable pairwise non-isomorphic ACM sheaves provided that the sheaves $\mathcal{F}$ are actually Ulrich.

Having this in mind, we consider the following set-up. Let $X \subset \mathbb{P}^{N}$ be a non-degenerate reduced closed ACM subscheme of dimension $n$ and degree $d$ over a field $\mathrm{k}$. Let $M \subset \mathbb{P}^{N}$ be a linear subspace of codimension $c \geqslant 1$, and assume that $Y=X \cap M$ is of dimension $n-c \geqslant 1$. Let $\mathcal{F}$ be an ACM sheaf on $Y$. By Section 4.1 and the discussion above, Theorem A holds for $d \geqslant N-n+2$ once we prove the following result.

TheOREM 4.4. The sheaf $\mathcal{E}=\Sigma_{c}^{X}(\mathcal{F})$ is Ulrich on $X$ if and only if $\mathcal{F}$ is Ulrich on $Y$ and $d=N-n+1$.

Proof. If $\mathcal{F}$ is Ulrich on $Y$ and $d=N-n+1$, then $\mathcal{E}$ is Ulrich on $X$ according to [FP15, Proposition 3.6]. What we have to prove is that $\mathcal{E}$ is not Ulrich on $X$ if either $\mathcal{F}$ is not Ulrich on $Y$ or $d \geqslant N-n+2$.

Put $R_{X}=\mathbb{k}[X]$ and $R_{Y}=\mathbb{k}[Y]$. We consider $F=\mathrm{H}_{*}^{0}(\mathcal{F})$ as a graded $R_{X}$-module. Recall that $F$ is a graded Cohen-Macaulay module on $R_{X}$, which is non-maximal as its dimension is $\operatorname{dim}\left(R_{Y}\right)=n-c+1<n+1=\operatorname{dim}\left(R_{X}\right)$. Without loss of generality, we may assume that $\mathcal{F}$ is initialized. So, the $R_{X}$-module $F$ is generated by finitely many elements of positive degree. Therefore, the free modules $\left(F_{i} \mid i \geqslant 0\right)$ appearing in the minimal graded free resolution $F$. of $F$ over $R_{X}$ take the form

$$
F_{i}=\bigoplus_{j \in\left\{i, \ldots, r_{i}\right\}} R_{X}(-j)^{\oplus a_{i, j}}
$$




\section{Faenzi, F. Malaspina and G. Sanna}

for some sequence of integers $\left(r_{i} \mid i \in \mathbb{N}\right)$ with $r_{i} \geqslant i$ for all $i \in \mathbb{N}$ and some uniquely determined integers $\left(a_{i, j} \mid i \in \mathbb{N}, j \in\left\{i, \ldots, r_{i}\right\}\right)$, called the $R_{X}$-Betti numbers of $F$.

Write the Koszul resolution $K$. of $R_{Y}$ over $R_{X}$ as

$$
0 \leftarrow R_{Y} \leftarrow K_{0} \leftarrow \cdots \leftarrow K_{c} \leftarrow 0 \text { with } K_{i}=R_{X}(-i)^{\oplus}\left(\begin{array}{c}
c \\
i
\end{array}\right) \text { for all } i \in\{0, \ldots, c\} .
$$

Since $\mathcal{F}$ is an initialized ACM sheaf over $Y$, there exists an injective map $\varphi: R_{Y} \rightarrow F$. This map lifts to a map of graded complexes $\varphi . K . \rightarrow F$., which we write, for $i \in\{0, \ldots, c\}$, as

$$
\varphi_{i}: \quad R_{X}(-i)^{\oplus\left(\begin{array}{l}
c \\
i
\end{array}\right)} \rightarrow \bigoplus_{j \in\left\{i, \ldots, r_{i}\right\}} R_{X}(-j)^{\oplus a_{i, j}} .
$$

In particular, the polynomial maps $\varphi_{0}, \ldots, \varphi_{c}$ are actually constant.

Next, we observe that $\varphi_{i}$ is injective for all $i \in\{0, \ldots, c\}$. Indeed, $\varphi_{0}$ is injective since $\varphi \neq 0$ and $K_{0}=R_{X}$. For $i \in\{1, \ldots, c\}$, by induction on $i$ we may assume that $\varphi_{i-1}$ is injective, so that the induced map $\Sigma_{i}^{X}\left(R_{Y}\right) \rightarrow \Sigma_{i}^{X}(F)$ is also injective. Thus $\operatorname{ker}\left(\varphi_{i}\right)$ is contained in $\Sigma_{i+1}^{X}\left(R_{Y}\right)$. But looking at (4.7), we see that $\operatorname{ker}\left(\varphi_{i}\right)$ is generated by elements of degree $i$, while all elements of $\Sigma_{i+1}^{X}\left(R_{Y}\right)$ have degree at least $i+1$. Therefore, $\operatorname{ker}\left(\varphi_{i}\right)=0$.

In view of the previous paragraph, for each $i \in\{0, \ldots, c\}$, we have a splitting $F_{i} \simeq K_{i} \oplus G_{i}$ for some graded $R_{X}$-module $G_{i}$ such that for each $i \in\{1, \ldots, c\}$, the differential $d_{i}: F_{i} \rightarrow F_{i-1}$ is upper triangular according to the block-matrix form

$$
d_{i}: K_{i} \oplus G_{i} \rightarrow K_{i-1} \oplus G_{i-1} ;
$$

that is, $K_{i} \rightarrow K_{i-1} \oplus G_{i-1}$ factors through the Koszul differential $K_{i} \rightarrow K_{i-1}$.

Thus the map $\varphi_{c-1}$ induces an injection

$$
R_{X}(-c)=\Sigma_{c}^{X}\left(R_{Y}\right) \hookrightarrow \Sigma_{c}^{X}(F)=E .
$$

This says that $\mathrm{H}^{0}(X, \mathcal{E}(c)) \neq 0$.

Next, use [FP15, sequence (3.2)] to get the long exact sequence

$$
0 \leftarrow \operatorname{Hom}_{R_{Y}}\left(F, R_{Y}(1)\right) \leftarrow E^{\vee}(1-c) \leftarrow F_{c-1}^{\vee}(1-c) \leftarrow \cdots \leftarrow F_{0}^{\vee}(1-c) \leftarrow 0 .
$$

Set $Q_{0}=F_{0}$ and for $i \in\{1, \ldots, c-1\}$, define

$$
Q_{i}=\operatorname{Coker}\left(F_{i-1}^{\vee}(1-c) \rightarrow F_{i}^{\vee}(1-c)\right),
$$

where the maps are extracted from the above complex. We get an injection $Q_{c-1} \rightarrow E^{\vee}(1-c)$. Note that the above maps have a block-matrix form which is the transpose of (4.8) and that the cokernel of the transpose of the Koszul differential $K_{c-2}^{\vee}(-c) \rightarrow K_{c-1}^{\vee}(-c)$ is the homogeneous ideal $I_{Y / X}$ of $Y$ in $X$. Therefore, the map $F_{c-2}^{\vee}(1-c) \rightarrow F_{c-1}^{\vee}(1-c)$ commutes with restricting the source to $K_{c-2}^{\vee}(1-c)$ and the target to $K_{c-1}^{\vee}(1-c)$. This gives an induced surjection $Q_{c-1} \rightarrow I_{Y / X}(1)$. Then, we may extract a non-trivial map

$$
R_{X}^{\oplus c}=K_{c-1}^{\vee}(1-c) \rightarrow Q_{c-1},
$$

which composes to a non-trivial map $R_{X}^{\oplus c} \rightarrow E^{\vee}(1-c)$. So $\mathrm{H}^{0}\left(X, \mathcal{E}^{\vee}(1-c)\right) \neq 0$.

Having set up all this, we can prove that if $d \geqslant N-n+2$, no integer $t$ turns $\mathcal{E}$ into an initialized Ulrich sheaf. Indeed, let $t$ be such an integer, so that $\mathrm{H}^{*}(X, \mathcal{E}(t-j))=0$ for $1 \leqslant j \leqslant n$. We proved $\mathrm{H}^{0}(X, \mathcal{E}(c)) \neq 0$ and hence $t \leqslant c$.

On the other hand, by [FP15, Lemma 3.1], since $X$ is not of minimal degree, we have that $\mathrm{H}^{0}\left(X, \omega_{X}(n-1)\right) \neq 0$. So there is an injective map $\mathcal{O}_{X} \rightarrow \omega_{X}(n-1)$. Tensoring $\mathcal{E}^{\vee}(1-c)$ with 


\section{NON-ULRICH REPRESENTATION TYPE}

this map and using $\mathrm{H}^{0}\left(X, \mathcal{E}^{\vee}(1-c)\right) \neq 0$, we get $\mathrm{H}^{0}\left(X, \mathcal{E}^{\vee} \otimes \omega_{X}(n-c)\right) \neq 0$. By Serre duality, we thus have $\mathrm{H}^{n}(X, \mathcal{E}(c-n)) \neq 0$, which implies $t \geqslant c+1$. Thus no integer $t$ turns $\mathcal{E}$ into an initialized Ulrich sheaf.

Finally, we prove that if $\mathcal{F}$ is not an initialized Ulrich sheaf on $Y$, then again no integer $t$ turns $\mathcal{E}(t)$ into an initialized Ulrich sheaf on $X$. By the previous argument, we have $t \leqslant c$, and we only need to prove $\mathrm{H}^{n}(X, \mathcal{E}(c-n)) \neq 0$. Now, if $\mathcal{F}$ is ACM but not Ulrich over the $(n-c)$ dimensional variety $Y$, since $\mathrm{H}^{0}(Y, \mathcal{F}(-1))=0 \neq \mathrm{H}^{0}(Y, \mathcal{F})$, we must have $\mathrm{H}^{n-c}(Y, \mathcal{F}(c-n)) \neq 0$. Sheafifying the resolution $F$. $\rightarrow F$ and taking cohomology, we get the following injections for $k \in\{1, \ldots, c\}:$

$$
\mathrm{H}^{n-c}(Y, \mathcal{F}(c-n)) \hookrightarrow \cdots \hookrightarrow \mathrm{H}^{n-c+k}\left(X, \Sigma_{k}^{X}(\mathcal{F})(c-n)\right) \hookrightarrow \cdots \hookrightarrow \mathrm{H}^{n}(X, \mathcal{E}(c-n)) .
$$

Therefore, $\mathrm{H}^{n}(X, \mathcal{E}(c-n)) \neq 0$. This concludes the proof.

\section{Varieties of minimal degree}

For this section, let $X \subset \mathbb{P}^{N}$ be a non-degenerate closed subscheme of dimension $n \geqslant 2$ over an algebraically closed field $\mathrm{k}$. Assume that $X$ is ACM of degree $d=N-n+1$; that is, $X$ is of minimal degree. We want to prove Theorem A for $X$.

First assume that $X$ is not integral (not reduced, or reduced but not irreducible). Then the argument of [FP15, Section 6] applies to show that $X$ is non-Ulrich CM-wild. This follows from the fact that $X$ supports ACM sheaves supported on the different components of $X$, or on the reduced structure of a component of $X$, and that we can twist the leftmost term of this extension by $\mathcal{O}_{X}(q)$ for $q \gg 0$ and therefore obtain a representation embedding whose source is a wild algebra and whose target consists of ACM sheaves on $X$ which are not Ulrich.

If $X$ is integral but not smooth, then $X$ is a cone over a smooth variety which is again ACM and of minimal degree, whose apex is a linear space of dimension $m \geqslant 0$. Also in this case, the argument of [FP15, Section 6.1] gives rise to ACM sheaves $\mathcal{E}$ which are not Ulrich except for finitely many choices of $\mathcal{E}$. Indeed, these sheaves arise as extensions of the form

$$
0 \rightarrow \mathcal{A}(q) \rightarrow \mathcal{E} \rightarrow \mathcal{B} \rightarrow 0,
$$

where $\mathcal{A}$ and $\mathcal{B}$ are sheaves obtained by extending to $X$ the presentation of initialized Ulrich sheaves on the base of the cone and $q>0$. For $q \gg 0$, the sheaf $\mathcal{E}$ cannot be Ulrich, so we get that $X$ is non-Ulrich CM-wild.

So from now on, in order to prove Theorem $\mathrm{A}$, we can assume that $X$ is smooth and irreducible; hence, according to the Bertini-del Pezzo classification (cf. [EH87]), the subscheme $X$ is a quadric hypersurface or a rational normal scroll.

After setting up some notation, this case will be settled in the next lemma. Given $n \geqslant 2$ and a non-decreasing sequence $a=\left(a_{1}, \ldots, a_{n}\right)$ of integers $1 \leqslant a_{1} \leqslant \cdots \leqslant a_{n}$, put $d=\sum_{i=1}^{n} a_{i}$ and $N=d+n-1$. We denote by $S(a)=S\left(a_{1}, \ldots, a_{n}\right)$ the rational normal scroll defined as the projectivization of $\oplus_{i=1}^{n} \mathcal{O}_{\mathbb{P}^{1}}\left(a_{i}\right)$, embedded as a submanifold of degree $d$ in $\mathbb{P}^{d+n-1}$ by the tautological relatively ample line bundle. We set $H$ for the hyperplane class of $S(a)$ and $F$ for the class of a fibre of the projection $S(a) \rightarrow \mathbb{P}^{1}$. Let $\mathcal{L}=\mathcal{O}_{X}((d-1) F-H)$.

It is well known that $X=S(a)$ is CM-finite if $a \in\{(1,1),(1,2)\}$. By [FM17], the scheme $X$ is CM-tame if $a \in\{(1,3),(2,2)\}$. We know by [Mir13] that the rational normal scroll $X=S(a)$ is Ulrich-wild except for the cases above, namely $a \in\{(1,1),(1,2),(1,3),(2,2)\}$. If we seek unbounded families of non-Ulrich bundles, we should be a bit more careful and exclude the excep- 


\section{Faenzi, F. Malaspina and G. Sanna}

tional cases appearing in the statement of Theorem A, which is to say $a \in\{(1,3),(2,2),(1,1,1)\}$.

So, from now on, we actually assume

$$
n \geqslant 4 \text { or } n=3, d \geqslant 4 \text { or } n=2, d \geqslant 5 .
$$

We start by noting that the set-up of [FM17, Theorem B] applies in any dimension to give rigid Ulrich bundles on $X$ associated with Fibonacci-like sequences. Indeed, put $\ell=(n-1) d-n$, so that $h^{i}\left(\mathcal{O}_{X}(H-d F)\right)=0$ for all $i \neq 1$ and hence by the Riemann-Roch theorem,

$$
\ell=-\chi\left(\mathcal{L}^{\vee}(-F)\right)=h^{1}\left(\mathcal{O}_{X}(H-d F)\right) \geqslant 2
$$

in our range for $(d, n)$. Define recursively the Fibonacci-like numbers $a_{\ell, k} \in \mathbb{N}$ by

$$
a_{\ell, 0}=0, \quad a_{\ell, 1}=1, \quad a_{\ell, k+2}=\ell a_{\ell, k+1}-a_{\ell, k} \quad \text { for } k \in \mathbb{N} .
$$

Since $\ell \geqslant 2$, the sequence $\left(a_{\ell, k}\right)$ is strictly increasing along $k$.

Recall the notion of exceptional sheaf $\mathcal{E}$ on $X$; namely, $\mathcal{E}$ is a simple coherent sheaf such that $\operatorname{Ext}_{X}^{i}(\mathcal{E}, \mathcal{E})=0$ for $i>0$. Also recall that two exceptional sheaves $(\mathcal{E}, \mathcal{F})$ form an exceptional pair if $\operatorname{Ext}_{X}^{i}(\mathcal{F}, \mathcal{E})=0$ for all $i$. The pair $\left(\mathcal{L}, \mathcal{O}_{X}(-F)\right)$ is exceptional. We mentioned that $h^{1}\left(\mathcal{L}^{\vee}(-F)\right)=\ell$ and $h^{i}\left(\mathcal{L}^{\vee}(-F)\right)=0$ for $i \neq 1$. Then, making use of mutations like in [FM17, Section 2], we get that for each $k \geqslant 0$, there is a unique exceptional sheaf $\mathcal{U}_{k}$ which fits into

$$
0 \rightarrow \mathcal{O}_{X}(-F)^{\oplus a_{\ell, k}} \rightarrow \mathcal{U}_{k} \rightarrow \mathcal{L}^{\oplus a_{\ell, k+1}} \rightarrow 0 .
$$

Theorem A will be proved for $X$ if we check the following result.

Lemma 5.1. The sheaves $\mathcal{B}=\mathcal{U}_{k}$ and $\mathcal{A}=\mathcal{O}_{X}$ satisfy the assumptions of Theorem 3.1 as long as we choose

$-k=0$ for $n \geqslant 4$,

$-k=1$ for $n=3$ and $d \geqslant 4$,

$-k=3$ for $n=2$ and $d \geqslant 5$.

Proof. Working as in [FM17, Section 2], we check that $\mathcal{U}_{k}$ is an exceptional Ulrich bundle which is actually initialized by $t=1$. As a consequence, $\mathcal{U}_{k}$ is (strictly) semistable simple sheaf with

$$
\mathrm{p}\left(\mathcal{U}_{k}\right)=\frac{t d}{n !} \prod_{i=1}^{n-1}(t+i) \prec \frac{t d+n}{n !} \prod_{i=1}^{n-1}(t+i)=\mathrm{p}\left(\mathcal{O}_{X}\right) .
$$

The sheaf $\mathcal{O}_{X}$ is not Ulrich and is obviously stable. So, in order to verify the assumptions of Theorem 3.1, it only remains to check the condition on the dimension of the extension space. We note that $h^{i}\left(\mathcal{L}^{\vee}\right)=0$ for $i \geqslant 2$ and that, by the Riemann-Roch theorem,

$$
\chi\left(\mathcal{L}^{\vee}\right)=2 n+(1-n) d .
$$

Looking at (5.1), we deduce $h^{i}\left(\mathcal{U}_{k}^{\vee}\right)=0$ for $i \geqslant 2$, so

$$
\begin{aligned}
\operatorname{dim}_{\mathbb{k}} \operatorname{Ext}_{X}^{1}\left(\mathcal{U}_{k}, \mathcal{O}_{X}\right) & =h^{1}\left(\mathcal{U}_{k}^{\vee}\right) \geqslant-\chi\left(\mathcal{U}_{k}^{\vee}\right) \\
& =-a_{\ell, k+1} \chi\left(\mathcal{L}^{\vee}\right)-2 a_{\ell, k}=a_{\ell, k+1}((n-1) d-2 n)-2 a_{\ell, k} .
\end{aligned}
$$

First, assume $n \geqslant 4$. In particular, we have $d \geqslant 4$. Recall that for the case $n \geqslant 4$, we have chosen $k=0$. Hence, we consider $\mathcal{U}_{0}=\mathcal{L}$ and note that $h^{1}\left(\mathcal{L}^{\vee}\right)=-\chi\left(\mathcal{L}^{\vee}\right) \geqslant 4$ because $(n-1) d-2 n \geqslant 3 d-8 \geqslant 4$. Thus, the lemma is proved for $n \geqslant 4$. Next, assume $n=3$, so that our choice is $k=1$. Then, formula (5.2) gives $h^{1}\left(\mathcal{U}_{1}^{\vee}\right) \geqslant 4 d^{2}-18 d+16$, which is at least 8 when 


\section{NON-ULRICH REPRESENTATION TYPE}

$d \geqslant 4$. Finally, let $n=2$, in which case we have chosen $k=3$. Then, evaluating (5.2), we get $h^{1}\left(\mathcal{U}_{3}^{\vee}\right) \geqslant d^{4}-10 d^{3}+32 d^{2}-36 d+10$. This is at least 5 for $d \geqslant 5$.

The proof of Theorem A is now complete.

\section{The Segre product of a line and a plane}

Let us now turn to the analysis of the Segre product $X=\mathbb{P}^{1} \times \mathbb{P}^{2}$, which we consider as a smooth submanifold of $\mathbb{P}^{5}$. In other words, $X$ is the rational normal scroll $X=S(1,1,1)$ of degree $d=3$ embedded by the tautological relatively ample divisor $H$, hence $X$ has minimal degree. A smooth hyperplane section of $X$ is the CM-finite cubic scroll $S(1,2)$.

Our goal here is to classify all ACM indecomposable bundles on $X$. Of course, this is not quite possible since Ulrich bundles form a wild class in terms of representation theory, so we focus on non-Ulrich bundles and we classify all those.

\subsection{A first classification result via homological non-vanishing}

Let us first give the basic ACM bundles that will be the output of the classification. Put $\pi$ for the projection $X \rightarrow \mathbb{P}^{1}$ and $\Omega_{\pi}$ for the relative cotangent bundle. Here $X$ is a product, so $\Omega_{\pi}$ is the pull-back of the cotangent bundle of $\mathbb{P}^{2}$ via the projection $\sigma: X \rightarrow \mathbb{P}^{2}$. Set $L=H-F$, so $\mathcal{O}_{X}(L)=\sigma^{*}\left(\mathcal{O}_{\mathbb{P}^{2}}(1)\right)$. Recall that we have

$$
\omega_{X} \simeq \mathcal{O}_{X}(-2 F-3 L) .
$$

We easily see that $\mathcal{O}_{X}(L)$ is ACM and $\Omega_{\pi}(H+L)$ is Ulrich. We start with a lemma, inspired by [BM11], that classifies these sheaves as bundles with a specific non-vanishing.

Lemma 6.1. Let $\mathcal{E}$ be a locally free sheaf on $X$. Then $\mathcal{E} \simeq \Omega_{\pi}(L)$ if and only if $\mathcal{E}$ is indecomposable and

$$
\mathrm{H}^{1}(\varepsilon)=\mathrm{H}^{1}(\mathcal{E}(-1))=\mathrm{H}^{2}(\mathcal{E}(-2))=0, \quad \mathrm{H}^{1}(\mathcal{E}(-L)) \neq 0 .
$$

Proof. One implication is clear, so we assume that $\mathcal{E}$ is an indecomposable locally free sheaf satisfying (6.1) and we prove that $\mathcal{E} \simeq \Omega_{\pi}(L)$. Recall the standard isomorphism $\operatorname{Ext}_{X}^{1}\left(\mathcal{O}_{X}(L), \mathcal{E}\right) \simeq$ $\mathrm{H}^{1}(\mathcal{E}(-L))$. Then, write the vertical Euler sequence

$$
0 \rightarrow \Omega_{\pi}(L) \rightarrow \mathcal{O}_{X}^{\oplus 3} \rightarrow \mathcal{O}_{X}(L) \rightarrow 0
$$

and apply $\operatorname{Hom}_{X}(-, \mathcal{E})$ to it. Since $\operatorname{Ext}_{X}^{1}\left(\mathcal{O}_{X}, \mathcal{E}\right)=\mathrm{H}^{1}(\mathcal{E})=0$, we get a surjection

$$
\operatorname{Hom}_{X}\left(\Omega_{\pi}(L), \mathcal{E}\right) \rightarrow \operatorname{Ext}_{X}^{1}\left(\mathcal{O}_{X}(L), \mathcal{E}\right) \simeq \mathrm{H}^{1}(\mathcal{E}(-L)) .
$$

Take $e \in \mathrm{H}^{1}(\mathcal{E}(-L)) \backslash\{0\}$, and consider a map $f: \Omega_{\pi}(L) \rightarrow \mathcal{E}$ lying in the preimage of $e$ under the above surjection.

Further, we consider the dual vertical Euler sequence, written in the form

$$
0 \rightarrow \mathcal{O}_{X}(-2 L) \rightarrow \mathcal{O}_{X}(-L)^{\oplus 3} \rightarrow \Omega_{\pi}(L) \rightarrow 0 .
$$

Note that, by Serre duality, our assumption gives

$$
\operatorname{Ext}_{X}^{1}\left(\mathcal{E}, \mathcal{O}_{X}(-L)\right) \simeq \mathrm{H}^{2}(\mathcal{E}(-2))^{\vee}=0 .
$$

Next, we write the horizontal Euler sequence in the form

$$
0 \rightarrow \mathcal{O}_{X}(-2 L-2 F) \rightarrow \mathcal{O}_{X}(-2 L-F)^{\oplus 2} \rightarrow \mathcal{O}_{X}(-2 L) \rightarrow 0 .
$$




\section{Faenzi, F. Malaspina and G. Sanna}

Again, our assumption gives, via Serre duality,

$$
\operatorname{Ext}_{X}^{2}\left(\mathcal{E}, \mathcal{O}_{X}(-2 L-F)\right) \simeq \mathrm{H}^{1}(\mathcal{E}(-1))^{\vee}=0 .
$$

We thus have a surjection as a composition of surjections:

$$
\operatorname{Hom}_{X}\left(\mathcal{E}, \Omega_{\pi}(L)\right) \rightarrow \operatorname{Ext}_{X}^{1}\left(\mathcal{E}, \mathcal{O}_{X}(-2 L)\right) \rightarrow \operatorname{Ext}_{X}^{2}\left(\mathcal{E}, \mathcal{O}_{X}(-2)\right) .
$$

Choose a generator $k_{X}$ of the vector space $\mathrm{H}^{3}\left(\omega_{X}\right)$ and $h \in \operatorname{Ext}_{X}^{2}\left(\mathcal{E}, \mathcal{O}_{X}(-2)\right)$ such that the Yoneda product

$$
\mathrm{H}^{1}(\mathcal{E}(-L)) \otimes \operatorname{Ext}_{X}^{2}\left(\mathcal{E}, \mathcal{O}_{X}(-2)\right) \rightarrow \mathrm{H}^{3}\left(\mathcal{O}_{X}(-2 F-3 L)\right) \simeq \mathrm{H}^{3}\left(\omega_{X}\right)
$$

sends $e \otimes h$ to $k$. Then, choose $g: \mathcal{E} \rightarrow \Omega_{\pi}(L)$ lying in the preimage of $h$ under the surjection (6.5).

It is well known that $\Omega_{\mathbb{P}^{2}}$ is a simple sheaf, so the same holds for $\Omega_{\pi}(L)$. Therefore, if the map $g \circ f$ is non-zero, it must be a non-zero multiple of the identity. This immediately implies that $\Omega_{\pi}(L)$ is a direct summand of $\mathcal{E}$, which forces $\mathcal{E} \simeq \Omega_{\pi}(L)$ because $\mathcal{E}$ is indecomposable.

It remains to check that $g \circ f \neq 0$. To do this, we consider the following commutative diagram of Yoneda maps:

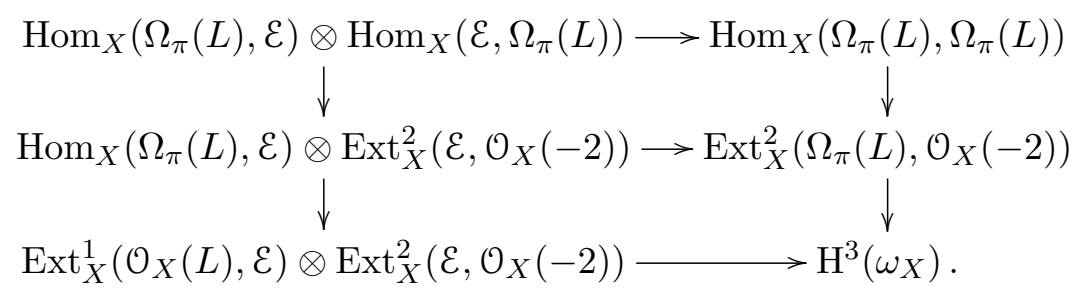

Our goal is to prove that the map appearing in the top row sends $f \otimes g$ to a non-zero element. The upper map in the left column sends $f \otimes g$ to $f \otimes h$, so it suffices to check that the map in the middle row sends $f \otimes h$ to a non-zero element. In turn, the lower map in the left column sends $f \otimes h$ to $e \otimes h$, so it is enough to show that the map in the bottom row sends $e \otimes h$ to a non-zero element. But this last map sends $e \otimes h$ to $k_{X}$; hence, we are done.

In a similar vein, we show the following.

Lemma 6.2. Let $\mathcal{E}$ be an indecomposable locally free sheaf on $X$. Then

i) there is an isomorphism $\mathcal{E} \simeq \mathcal{O}_{X}(-L)$ if and only if

$$
\mathrm{H}^{0}(\mathcal{E})=\mathrm{H}^{1}(\mathcal{E}(-L))=\mathrm{H}^{2}(\mathcal{E}(-F-2 L))=0, \quad \mathrm{H}^{0}(\mathcal{E}(L)) \neq 0 ;
$$

ii) there is an isomorphism $\mathcal{E} \simeq \mathcal{O}_{X}(-1)$ if and only if

$$
\mathrm{H}^{0}(\mathcal{E}(L))=\mathrm{H}^{1}(\mathcal{E}(-F))=\mathrm{H}^{2}(\mathcal{E}(-1))=0, \quad \mathrm{H}^{0}(\mathcal{E}(1)) \neq 0 .
$$

Proof. Both items have an obvious implication, what we have to prove is that $\mathcal{E}$ is isomorphic to the desired sheaf after assuming the cohomological conditions.

Let us prove item i). Choose a non-zero element $f$ of $\mathrm{H}^{0}(\mathcal{E}(L)) \simeq \operatorname{Hom}_{X}\left(\mathcal{O}_{X}(-L), \mathcal{E}\right)$. Next, we choose a generator $k_{X}$ of $\mathrm{H}^{3}\left(\omega_{X}\right)$ and note that, by Serre duality, there exists an $h \in \operatorname{Ext}_{X}^{3}\left(\mathcal{E}, \mathcal{O}_{X}(-2 F-4 L)\right)$ such that the Yoneda pairing

$$
\operatorname{Hom}_{X}\left(\mathcal{O}_{X}(-L), \mathcal{E}\right) \otimes \operatorname{Ext}_{X}^{3}\left(\mathcal{E}, \mathcal{O}_{X}(-2 F-4 L)\right) \rightarrow \mathrm{H}^{3}\left(\omega_{X}\right)
$$

sends $f \otimes h$ to $k$. 


\section{NON-ULRICH REPRESENTATION TYPE}

Next, write the exact sequences (6.2), (6.3) and (6.4) again, twisted by lines bundles on $X$ so that they take the following form:

$$
\begin{aligned}
& 0 \rightarrow \mathcal{O}_{X}(-2 F-4 L) \rightarrow \mathcal{O}_{X}(-2 F-3 L)^{\oplus 3} \rightarrow \Omega_{\pi}(-2 F-L) \rightarrow 0, \\
& 0 \rightarrow \Omega_{\pi}(-2 F-L) \rightarrow \mathcal{O}_{X}(-2)^{\oplus 3} \rightarrow \mathcal{O}_{X}(-2 F-L) \rightarrow 0, \\
& 0 \rightarrow \mathcal{O}_{X}(-2 F-L) \rightarrow \mathcal{O}_{X}(-1)^{\oplus 2} \rightarrow \mathcal{O}_{X}(-L) \rightarrow 0 .
\end{aligned}
$$

We remark that the vanishing assumptions of item i) and Serre duality imply

$$
\begin{aligned}
& \operatorname{Ext}_{X}^{3}\left(\mathcal{E}, \mathcal{O}_{X}(-2 F-3 L)\right) \simeq \mathrm{H}^{0}(\mathcal{E})^{*}=0, \\
& \operatorname{Ext}_{X}^{2}\left(\mathcal{E}, \mathcal{O}_{X}(-2)\right) \simeq \mathrm{H}^{1}(\mathcal{E}(-L))^{*}=0, \\
& \operatorname{Ext}_{X}^{1}\left(\mathcal{E}, \mathcal{O}_{X}(-1)\right) \simeq \mathrm{H}^{2}(\mathcal{E}(-F-2 L))^{*}=0 .
\end{aligned}
$$

Therefore, applying $\operatorname{Hom}_{X}(\varepsilon,-)$ to the three sequences above, we get a surjection

$$
\operatorname{Hom}_{X}\left(\mathcal{E}, \mathcal{O}_{X}(-L)\right) \rightarrow \operatorname{Ext}_{X}^{3}\left(\mathcal{E}, \mathcal{O}_{X}(2 F-4 L)\right)
$$

We now choose $g \in \operatorname{Hom}_{X}\left(\mathcal{E}, \mathcal{O}_{X}(-L)\right)$ in the preimage of $h$.

Therefore, we have a commutative diagram of the form

$$
\begin{aligned}
\operatorname{Hom}_{X}\left(\mathcal{O}_{X}(-L), \mathcal{E}\right) \otimes \operatorname{Hom}_{X}\left(\mathcal{E}, \mathcal{O}_{X}(-L)\right) \longrightarrow & \operatorname{Hom}_{X}\left(\mathcal{O}_{X}(-L), \mathcal{O}_{X}(-L)\right) \\
& \downarrow \\
\operatorname{Hom}_{X}\left(\mathcal{O}_{X}(-L), \mathcal{E}\right) \otimes & \operatorname{Ext}_{X}^{1}\left(\mathcal{E}, \mathcal{O}_{X}(-2 F-L)\right) \longrightarrow \operatorname{Ext}_{X}^{1}\left(\mathcal{O}_{X}(-L), \mathcal{O}_{X}(-2 F-L)\right) \\
& \downarrow \\
& \\
\operatorname{Hom}_{X}\left(\mathcal{O}_{X}(-L), \mathcal{E}\right) \otimes & \operatorname{Ext}_{X}^{2}\left(\mathcal{E}, \Omega_{\pi}(-2 F-L)\right) \longrightarrow \operatorname{Ext}_{X}^{2}\left(\mathcal{O}_{X}(-L), \Omega_{\pi}(-2 F)\right) \\
& \downarrow \\
& \downarrow \\
\operatorname{Hom}_{X}\left(\mathcal{O}_{X}(-L), \mathcal{E}\right) \otimes & \operatorname{Ext}_{X}^{3}\left(\mathcal{E}, \mathcal{O}_{X}(2 F-4 L)\right) \rightarrow \operatorname{Ext}^{3}\left(\mathcal{O}_{X}(-L), \mathcal{O}_{X}(-2 F-4 L)\right),
\end{aligned}
$$

where the horizontal maps are given by the Yoneda pairing, the left vertical ones are given by the factorization of the map (6.9), while the maps in the right vertical column are obtained by applying $\operatorname{Hom}_{X}\left(\mathcal{O}_{X}(-L),-\right)$ to the three exact sequences above. Since the identity map of $\mathcal{O}_{X}(-L)$ is sent to $k_{X}$ via the composition of vertical maps by construction, it follows that $g \circ f$ is sent to the identity of $\mathcal{O}_{X}(-L)$ via the top horizontal map. This says that $\mathcal{O}_{X}(-L)$ is a direct summand of $\mathcal{E}$ and therefore proves $\mathcal{E} \simeq \mathcal{O}_{X}(-L)$ by the indecomposability of $\mathcal{E}$.

The proof of item ii) is similar, so we only sketch the argument. The strategy this time is to apply $\operatorname{Hom}_{X}(\mathcal{E},-)$ to the exact sequences

$$
\begin{aligned}
& 0 \rightarrow \mathcal{O}_{X}(-3 F-4 L) \rightarrow \mathcal{O}_{X}(-2 F-4 L)^{\oplus 2} \rightarrow \mathcal{O}_{X}(-F-4 L) \rightarrow 0, \\
& 0 \rightarrow \mathcal{O}_{X}(-F-4 L) \rightarrow \mathcal{O}_{X}(-F-3 L)^{\oplus 3} \rightarrow \Omega_{\pi}(-1) \rightarrow 0, \\
& 0 \rightarrow \Omega_{\pi}(-1) \rightarrow \mathcal{O}_{X}(-F-2 L)^{\oplus 3} \rightarrow \mathcal{O}_{X}(-1) \rightarrow 0
\end{aligned}
$$

and to use Serre duality which gives, via the assumption of item ii),

$$
\begin{aligned}
& \operatorname{Ext}_{X}^{3}\left(\mathcal{E}, \mathcal{O}_{X}(-2 F-4 L)\right) \simeq \mathrm{H}^{0}(\mathcal{E}(L))^{*}=0, \\
& \operatorname{Ext}_{X}^{2}\left(\mathcal{E}, \mathcal{O}_{X}(-F-3 L)\right) \simeq \mathrm{H}^{1}(\mathcal{E}(-F))^{*}=0, \\
& \operatorname{Ext}_{X}^{1}\left(\mathcal{E}, \mathcal{O}_{X}(-F-2 L)\right) \simeq \mathrm{H}^{2}(\mathcal{E}(-1))^{*}=0 .
\end{aligned}
$$

The rest of the proof follows the same pattern as that of item i). 


\section{Faenzi, F. Malaspina and G. Sanna}

\subsection{Beilinson-type spectral sequence}

We use the derived category $\mathrm{D}(X)$ of bounded complexes of coherent sheaves over the smooth projective variety $X$ in order to write the Beilinson-type spectral sequence associated with a coherent sheaf $\mathcal{E}$ on $X$ after fixing a convenient full exceptional sequence in $\mathrm{D}(X)$. Indeed, the point is that the terms of this spectral sequence take a special form when $\mathcal{E}$ is ACM, and this will be our basic tool to classify such sheaves.

6.2.1 Background on exceptional objects and mutations. Let us first recall some terminology. An object $\mathcal{E}$ of $\mathrm{D}(X)$ is called exceptional if $\operatorname{Ext}_{X}^{\bullet}(\mathcal{E}, \mathcal{E})=\mathbb{k}$, concentrated in degree zero. An ordered set of exceptional objects $\left(\mathcal{E}_{0}, \ldots, \mathcal{E}_{s}\right)$ is called an exceptional collection if $\operatorname{Ext}_{X}^{\bullet}\left(\mathcal{E}_{i}, \mathcal{E}_{j}\right)=0$ for $i>j$. An exceptional collection is full when $\operatorname{Ext}_{X}^{\bullet}\left(\mathcal{E}_{i}, \mathcal{F}\right)=0$ for all $i$ implies $\mathcal{F}=0$. Equivalently, the collection is full when $\operatorname{Ext}_{X}^{\bullet}\left(\mathcal{F}, \mathcal{E}_{i}\right)=0$ implies $\mathcal{F}=0$.

Exceptional collections can be mutated; let us recall what that means. Let $\mathcal{E}$ be an exceptional object in $\mathrm{D}(X)$. Then there are endofunctors $\mathrm{L}_{\mathcal{E}}$ and $\mathrm{R}_{\mathcal{\varepsilon}}$ of $\mathrm{D}(X)$, called respectively the left and right mutation functors, such that for all $\mathcal{F}$ in $\mathrm{D}(X)$, there are functorial distinguished triangles

$$
\begin{aligned}
& \mathrm{L}_{\mathcal{E}}(\mathcal{F}) \rightarrow \operatorname{Ext}_{X}^{\bullet}(\mathcal{E}, \mathcal{F}) \otimes \mathcal{E} \rightarrow \mathcal{F} \rightarrow \mathrm{L}_{\mathcal{E}}(\mathcal{F})[1], \\
& \mathrm{R}_{\mathcal{E}}(\mathcal{F})[-1] \rightarrow \mathcal{F} \rightarrow \operatorname{Ext}_{X}^{\bullet}(\mathcal{F}, \mathcal{E})^{\vee} \otimes \mathcal{E} \rightarrow \mathrm{R}_{\mathcal{E}}(\mathcal{F}) .
\end{aligned}
$$

For all $i=0, \ldots, s$, we define the right and left dual objects

$$
\mathcal{E}_{i}^{\mathrm{d}}=\mathrm{L}_{\mathcal{E}_{0}} \mathrm{~L}_{\mathcal{E}_{1}} \cdots \mathrm{L}_{\mathcal{E}_{s-i-1}} \mathcal{E}_{s-i}, \quad{ }^{\mathrm{d}} \mathcal{E}_{i}=\mathrm{R}_{\mathcal{E}_{s}} \mathrm{R}_{\mathcal{E}_{s-1}} \cdots \mathrm{R}_{\mathcal{E}_{s-i+1}} \mathcal{E}_{s-i} .
$$

It turns out that if $\mathcal{E}_{\bullet}=\left(\varepsilon_{0}, \ldots, \mathcal{E}_{s}\right)$ is a full exceptional collection, then both $\left(\varepsilon_{0}^{\mathrm{d}}, \ldots, \mathcal{E}_{s}^{\mathrm{d}}\right)$ and ${ }^{\mathrm{d}} \mathcal{E}_{\bullet}=\left({ }^{\mathrm{d}} \varepsilon_{0}, \ldots,{ }^{\mathrm{d}} \mathcal{E}_{s}\right)$ also are full exceptional collections, called respectively the right and left dual collections of $\left(\mathcal{E}_{0}, \ldots, \mathcal{E}_{s}\right)$. We refer to [GK04, Section 2.6]. The dual collections are characterized by the following property:

$$
\operatorname{Ext}_{X}^{\ell}\left({ }^{\mathrm{d}} \mathcal{E}_{i}, \mathcal{E}_{j}\right) \simeq \operatorname{Ext}_{X}^{\ell}\left(\mathcal{E}_{i}, \mathcal{E}_{j}^{\mathrm{d}}\right) \simeq \begin{cases}\mathrm{k} & \text { if } i+j=s \text { and } i=\ell, \\ 0 & \text { otherwise }\end{cases}
$$

Given an object $\mathcal{F}$ of $\mathrm{D}(X)$ and a full exceptional collection $\left(\mathcal{E}_{0}, \ldots, \mathcal{E}_{s}\right)$, there is a spectral sequence

$$
\bigoplus_{r+t=q} \operatorname{Ext}_{X}^{r}\left({ }^{\mathrm{d}} \mathcal{E}_{s-p}, \mathcal{F}\right) \otimes \mathcal{H}^{t}\left(\mathcal{E}_{p}\right)=E_{1}^{p, q} \Rightarrow \mathcal{H}^{p+q-s}(\mathcal{F})
$$

where $\mathcal{H}^{i}$ denotes the $i$ th homology sheaf of $\mathcal{F}$. This means that for all $(p, q)$ such that $p+q \neq s$, we have $E_{\infty}^{p, q}=0$, while

$$
\bigoplus_{p+q=s} E_{\infty}^{p, q} \simeq \operatorname{gr}(\mathcal{F})
$$

where $\operatorname{gr}(\mathcal{F})$ denotes the graded object with respect to a filtration of $\mathcal{F}$ of the form

$$
\mathcal{F}=\mathcal{F}_{0} \supset \mathcal{F}_{1} \supset \cdots \supset \mathcal{F}_{s} \supset \mathcal{F}_{s+1}=0 \text { with } \mathcal{F}_{j} / \mathcal{F}_{j+1} \simeq E_{\infty}^{j, s-j} .
$$

The $r$ th differential of the spectral sequence reads $\delta_{r}^{p, q}: E_{r}^{p, q} \rightarrow E_{r}^{p+r, q-r+1}$.

6.2.2 An exceptional collection adapted to ACM sheaves. Let us choose a full exceptional collection over $X$ adapted to the classification of ACM sheaves. Recall that we denoted by $F$ the divisor class of the $\mathbb{P}^{2}$-bundle map $\pi: X \rightarrow \mathbb{P}^{1}$ such that $\mathrm{D}(X)$ has the following semiorthogonal 


\section{NON-ULRICH REPRESENTATION TYPE}

decomposition:

$$
\begin{aligned}
\mathrm{D}(X) & =\left\langle\pi^{*} \mathrm{D}\left(\mathbb{P}^{1}\right) \otimes \mathcal{O}_{X}(-2 L), \pi^{*} \mathrm{D}\left(\mathbb{P}^{1}\right) \otimes \mathcal{O}_{X}(-L), \pi^{*} \mathrm{D}\left(\mathbb{P}^{1}\right)\right\rangle \\
& =\left\langle\mathcal{O}_{X}(-F-2 L), \mathcal{O}_{X}(-2 L), \mathcal{O}_{X}(-F-L), \mathcal{O}_{X}(-L), \mathcal{O}_{X}(-F), \mathcal{O}_{X}\right\rangle .
\end{aligned}
$$

Twisting by a line bundle $\mathcal{O}_{X}(L)$ and performing a right mutation given by the Euler sequences on $\mathbb{P}^{1}$, this is replaced by

$$
\mathrm{D}(X)=\left\langle\mathcal{O}_{X}(-L), \mathcal{O}_{X}(F-L), \mathcal{O}_{X}(-F), \mathcal{O}_{X}, \mathcal{O}_{X}(L-F), \mathcal{O}_{X}(L)\right\rangle .
$$

Since $\mathcal{O}_{X}$ and $\mathcal{O}_{X}(L-F)$ are mutually orthogonal, mutation gives

$$
\mathrm{D}(X)=\left\langle\left(\mathcal{O}_{X}(-L), \mathcal{O}_{X}(F-L), \mathcal{O}_{X}(-F), \mathcal{O}_{X}(L-F), \mathcal{O}_{X}, \mathcal{O}_{X}(L)\right\rangle .\right.
$$

Finally, a right mutation given by the Euler sequences on $\mathbb{P}^{2}$ gives the following full exceptional collection of vector bundles over $X$ :

$$
\mathcal{E}_{\bullet}=\left(\mathcal{O}_{X}(-L), \mathcal{O}_{X}(F-L), \mathcal{O}_{X}(-F), \mathcal{O}_{X}(L-F), \Omega_{\pi}(L), \mathcal{O}_{X}\right) .
$$

Setting $\mathcal{T}_{\pi}=\Omega_{\pi}^{\vee} \simeq \Omega_{\pi}(3 L)$, we write the left dual of this collection as

$$
\mathrm{d} \mathcal{E}_{\bullet}=\left(\mathcal{O}_{X}, \mathcal{O}_{X}(L), \mathcal{O}_{X}(1)[1], \mathcal{T}_{\pi}(F)[1], \mathcal{O}_{X}(F+2 L)[2], \mathcal{O}_{X}(2)[2]\right) .
$$

Note that $\mathcal{E}_{1}=\mathcal{O}_{X}(F-L)$ is the Ulrich line bundle $\mathcal{L}$ from Section 5. By Künneth's formula, one gets another special feature of this collection, namely that

$$
\begin{aligned}
& \operatorname{Hom}_{X}\left(\varepsilon_{0}, \varepsilon_{2}\right)=\operatorname{Hom}_{X}\left(\varepsilon_{0}, \varepsilon_{3}\right)=0, \\
& \operatorname{Hom}_{X}\left(\varepsilon_{1}, \varepsilon_{j}\right)=0 \text { for } j \neq 1, \\
& \operatorname{Hom}_{X}\left(\varepsilon_{2}, \varepsilon_{4}\right)=0, \\
& \operatorname{Hom}_{X}\left(\varepsilon_{3}, \varepsilon_{4}\right)=0 .
\end{aligned}
$$

\subsection{Beilinson resolution of non-Ulrich sheaves}

Our goal for this subsection is to prove the next result.

Theorem 6.3. Up to a twist by $\mathcal{O}_{X}(t)$, an indecomposable $A C M$ bundle $\mathcal{F}$ on $X$ is isomorphic to $\mathcal{O}_{X}(-1)$, or to $\mathcal{O}_{X}(-L)$, or to $\Omega_{\pi}(L)$, or to an Ulrich bundle $\mathcal{U}$ fitting into

$$
0 \rightarrow \mathcal{O}_{X}(-F)^{\oplus a} \rightarrow \mathcal{U} \rightarrow \mathcal{O}_{X}(F-L)^{\oplus b} \rightarrow 0 \quad \text { for some }(a, b) \in \mathbb{N}^{2} .
$$

The words "up to a twist" have the following more precise meaning: up to replacing $\mathcal{F}$ with $\mathcal{F}(t)$, we may assume that $h^{0}(\mathcal{F})=0$ and $h^{0}(\mathcal{F}(1)) \neq 0$. Then $\mathcal{F}$ is exactly one of the sheaves appearing in the statement of Theorem 6.3. In other words, Theorem 6.3 proves Theorem B from the introduction.

We will prove the theorem through several claims. The very first argument is to use Lemma 6.1. Note that the vanishing conditions appearing in that lemma are verified for any twist of $\mathcal{F}$ since $\mathcal{F}$ is ACM, so if there is a twist $t \in \mathbb{Z}$ such that $\mathrm{H}^{1}(\mathcal{F}(t H-L)) \neq 0$, we will have $\mathcal{F}(t) \simeq \Omega_{\pi}(L)$. Theorem 6.3 is proved in this case.

Therefore, from now on we may assume $\mathrm{H}^{1}(\mathcal{F}(t H-L))=0$ for all $t \in \mathbb{Z}$. The next step is to observe that since $\mathcal{F}$ locally free and $\mathcal{O}_{X}(1)$ is very ample, there is a unique $t_{0} \in \mathbb{Z}$ such that $\mathcal{F}\left(t_{0}\right)$ satisfies $h^{0}\left(\mathcal{F}\left(t_{0}\right)\right)=0$ and $h^{0}\left(\mathcal{F}\left(t_{0}+1\right)\right) \neq 0$. We implicitly replace $\mathcal{F}$ with $\mathcal{F}\left(t_{0}\right)$ from now on. In particular, we have $\mathrm{H}^{0}(X, \mathcal{F})=0$. We put

$$
a_{i, j}=\operatorname{dim}_{\mathbb{k}} \operatorname{Ext}_{X}^{i}\left({ }^{\mathrm{d}} \mathcal{E}_{j}, \mathcal{F}\right) .
$$




\section{Faenzi, F. Malaspina and G. Sanna}

Claim 6.4. Let $\mathcal{F}$ be as above. Then $a_{1,3}=a_{2,4}=a_{2,3}=a_{3,4}=0$.

Proof. Recall that in view of Lemma 6.1, we may assume $\mathrm{H}^{1}(\mathcal{F}(-L))=0$. Let us summarize the vanishing conditions we have so far by writing down the matrix $\left(a_{i, j}\right)$. Traditionally, one rather writes the table $\left(b_{i, j}\right)=\left(a_{5-i, 5-j}\right)$ :

\begin{tabular}{|c|c|c|c|c|c|}
\hline $\mathcal{F}(-2)[-2]$ & $\mathcal{F}(-F-2 L)[-2]$ & $\mathcal{F} \otimes \Omega_{\pi}(-F)[-1]$ & $\mathcal{F}(-1)[-1]$ & $\mathcal{F}(-L)$ & $\mathcal{F}$ \\
\hline \hline$a_{5,5}$ & $a_{5,4}$ & 0 & 0 & 0 & 0 \\
\hline 0 & $a_{4,4}$ & $a_{4,3}$ & $a_{4,2}$ & 0 & 0 \\
\hline 0 & $a_{3,4}$ & $a_{3,3}$ & 0 & $a_{3,1}$ & $a_{3,0}$ \\
\hline 0 & $a_{2,4}$ & $a_{2,3}$ & 0 & $a_{2,1}$ & 0 \\
\hline 0 & 0 & $a_{1,3}$ & 0 & 0 & 0 \\
\hline 0 & 0 & 0 & 0 & 0 & 0 \\
\hline \hline $\mathcal{O}_{X}(-L)$ & $\mathcal{O}_{X}(F-L)$ & $\mathcal{O}_{X}(-F)$ & $\mathcal{O}_{X}(L-F)$ & $\Omega_{\pi}(L)$ & $\mathcal{O}_{X}$ \\
\hline
\end{tabular}

This table means that the $(p, q)$ th term of $E_{1}^{p, q}$ is the direct sum of as many copies of $\mathcal{E}_{i}$ as the coefficient $\left(b_{i, j}\right)$ appearing in the above table. Also, the coefficients above are obtained by computing the dimension of the cohomology of the bundle appearing in the $i$ th column of the first row, reading cohomological degree from bottom to top, with a shift indicated by the brackets.

Let us focus on the summand $\mathcal{O}_{X}(F-L)^{\oplus a_{2,4}}=E_{1}^{1,2}$. By (6.12), we have $\delta_{1}^{1,2}=0$. Obviously, $\delta_{r}^{1,2}=0$ for $r \geqslant 2$. Also, $E_{1}^{p, q}=0$ for $p+q \leqslant 2$, so $\mathcal{O}_{X}(F-L)^{\oplus a_{2,4}}$ survives to $E_{\infty}^{1,2}$, which in turn is zero because $E_{\infty}^{p, q}$ is concentrated at $p+q=5$. Therefore, $a_{2,4}=0$. For the same reason, we get $a_{1,3}=0$. Summing up, $E_{1}^{p, q}=0$ for $p+q \leqslant 3$.

Let us now look at the summand $\mathcal{O}_{X}(-F)^{\oplus a_{2,3}}=E_{1}^{2,2}$. The map $\delta_{r}^{2,2}$ is clearly zero for all $r \geqslant 1$, and since we proved $E_{1}^{p, q}=0$ for $p+q \leqslant 3$, we get $a_{2,3}=0$ again because $E_{\infty}^{p, q}$ is concentrated at $p+q=5$. The last vanishing $a_{3,4}=0$ follows a similar pattern.

In terms of the Beilinson spectral sequence, the previous claim shows $E_{1}^{p, q}=0$ for $p+q \leqslant 4$. Because of (6.12), we have $\delta_{r}^{2,3}=0$ for all $r \geqslant 1$, so the vanishing of $E_{1}^{p, q}$ with $p+q \leqslant 4$ implies that the term $\mathcal{O}_{X}(-F)^{\oplus a_{3,3}}$ survives at $E_{\infty}^{2,3}$ and is thus a direct summand of $\operatorname{gr}(\mathcal{F})$. For the same reason, $\mathcal{O}_{X}(F-L)^{\oplus a_{4,4}}$ survives at $E_{\infty}^{1,4}$. This means that the filtration of $\mathcal{F}$ induced by the Beilinson-type spectral sequence takes the form

$$
0=\mathcal{F}_{6} \subset \mathcal{F}_{5} \subset \cdots \subset \mathcal{F}_{0}=\mathcal{F}
$$

with

$$
\mathcal{F}_{5}=\mathcal{F}_{4}=\mathcal{F}_{3}=0, \quad \mathcal{F}_{2} \simeq \mathcal{O}_{X}(-F)^{\oplus a_{3,3}}, \quad \mathcal{F}_{1} / \mathcal{F}_{2} \simeq \mathcal{O}_{X}(F-L)^{\oplus a_{4,4}}, \quad \mathcal{F} / \mathcal{F}_{1} \simeq E_{\infty}^{0,5} .
$$

Our next goal is to compute $E_{\infty}^{0,5}$.

Lemma 6.5. There is an exact sequence

$$
0 \rightarrow E_{\infty}^{0,5} \rightarrow \mathcal{G} \rightarrow \Omega_{\pi}(L)^{\oplus a_{2,1}} \rightarrow 0,
$$

where $\mathcal{G}$ is a coherent sheaf on $X$ fitting into a long exact sequence

$$
\begin{aligned}
0 \rightarrow \mathcal{G} & \rightarrow \operatorname{Ker}\left(\delta_{1}^{0,5}\right) \rightarrow \operatorname{Ker}\left(\delta_{1}^{2,4}\right) \rightarrow \operatorname{Ker}\left(\delta_{1}^{4,3}\right) \\
& \rightarrow \operatorname{Coker}\left(\delta_{1}^{0,5}\right) \rightarrow \operatorname{Coker}\left(\delta_{1}^{2,4}\right) \rightarrow \operatorname{Coker}\left(\delta_{1}^{4,3}\right) \rightarrow 0 .
\end{aligned}
$$




\section{Non-UlRich REPRESENTATION TYPE}

Before going into the proof, let us display the maps $\delta_{1}^{p, q}$ we are interested in

$$
\begin{aligned}
& \delta_{1}^{0,5}: \mathcal{O}_{X}(-L)^{\oplus a_{5,5}} \rightarrow \mathcal{O}_{X}(F-L)^{\oplus a_{5,4}}, \\
& \delta_{1}^{2,4}: \mathcal{O}_{X}(-F)^{\oplus a_{4,3}} \rightarrow \mathcal{O}_{X}(L-F)^{\oplus a_{4,2}}, \\
& \delta_{1}^{4,3}: \Omega_{\pi}(L)^{\oplus a_{3,1}} \rightarrow \mathcal{O}_{X}^{\oplus a_{3,0}} .
\end{aligned}
$$

Proof. We rewrite the cohomology table $\left(b_{i, j}\right)$ in view of the vanishing proved in the previous claim and after removing $a_{3,3}$ and $a_{4,4}$, which do not contribute to $E_{\infty}^{0,5}$, as we have just seen.

\begin{tabular}{|c|c|c|c|c|c|}
\hline $\mathcal{F}(-2)[-2]$ & $\mathcal{F}(-F-2 L)[-2]$ & $\mathcal{F} \otimes \Omega_{\pi}(-F)[-1]$ & $\mathcal{F}(-1)[-1]$ & $\mathcal{F}(-L)$ & $\mathcal{F}$ \\
\hline \hline$a_{5,5}$ & $a_{5,4}$ & 0 & 0 & 0 & 0 \\
\hline 0 & 0 & $a_{4,3}$ & $a_{4,2}$ & 0 & 0 \\
\hline 0 & 0 & 0 & 0 & $a_{3,1}$ & $a_{3,0}$ \\
\hline 0 & 0 & 0 & 0 & $a_{2,1}$ & 0 \\
\hline 0 & 0 & 0 & 0 & 0 & 0 \\
\hline 0 & 0 & 0 & 0 & 0 & 0 \\
\hline \hline $\mathcal{O}_{X}(-L)$ & $\mathcal{O}_{X}(F-L)$ & $\mathcal{O}_{X}(-F)$ & $\mathcal{O}_{X}(L-F)$ & $\Omega_{\pi}(L)$ & $\mathcal{O}_{X}$ \\
\hline
\end{tabular}

In view of this table, we see that the differential $\delta_{1}$ has only three possibly non-zero terms, namely $\delta_{1}^{0,5}, \delta_{1}^{2,4}$ and $\delta_{1}^{4,3}$. So $E_{2}^{p, q}$ differs from $E_{1}^{p, q}$ only when $(p, q)$ equals $(0,5),(1,5),(2,4)$, $(3,4),(4,3)$ and $(5,3)$, and we get

$$
\begin{array}{ll}
E_{2}^{0,5} \simeq \operatorname{ker}\left(\delta_{1}^{0,5}\right), & E_{2}^{1,5} \simeq \operatorname{Coker}\left(\delta_{1}^{0,5}\right), \\
E_{2}^{2,4} \simeq \operatorname{ker}\left(\delta_{1}^{2,4}\right), & E_{2}^{3,4} \simeq \operatorname{Coker}\left(\delta_{1}^{2,4}\right), \\
E_{2}^{4,3} \simeq \operatorname{ker}\left(\delta_{1}^{4,3}\right), & E_{2}^{5,3} \simeq \operatorname{Coker}\left(\delta_{1}^{4,3}\right) .
\end{array}
$$

Now, since $E_{\infty}^{p, q}$ is concentrated at $p+q=5$, we realize that actually $E_{3}^{5,3}=0$, so the map $\delta_{2}^{3,4}: E_{2}^{3,4} \rightarrow E_{2}^{5,3}$ is surjective and actually also $E_{3}^{3,4}=0$; hence, the kernel of $\delta_{2}^{3,4}$ is the image of $\delta_{2}^{0,5}$. We have thus proved the second line of (6.15). For the same reason, we have the exactness of the sequence

$$
\operatorname{Ker}\left(\delta_{1}^{0,5}\right) \rightarrow \operatorname{Ker}\left(\delta_{1}^{2,4}\right) \rightarrow \operatorname{Ker}\left(\delta_{1}^{4,3}\right),
$$

where the maps are just $\delta_{2}^{0,5}$ and $\delta_{2}^{2,4}$.

This completes the analysis of the second page of the spectral sequence. We turn now to $E_{3}$. Note that $E_{3}^{1,5} \simeq \operatorname{Ker}\left(\delta_{2}^{1,5}\right)$ is the kernel of the map $\delta_{2}^{1,5}: \operatorname{Coker}\left(\delta_{1}^{0,5}\right) \rightarrow \operatorname{Coker}\left(\delta_{1}^{2,4}\right)$ appearing in (6.15). Similarly, $E_{3}^{4,3} \simeq \operatorname{Coker}\left(\delta_{2}^{2,4}\right)$ is the cokernel of the map $\delta_{2}^{2,4}: \operatorname{Ker}\left(\delta_{1}^{2,4}\right) \rightarrow \operatorname{Ker}\left(\delta_{1}^{4,3}\right)$ showing up in (6.19). Since $E_{4}^{1,5}=E_{4}^{4,3}=0$, the map $\delta_{3}^{1,5}$ gives an isomorphism from $E_{3}^{1,5}$ to $E_{3}^{4,3}$; hence, the exactness of (6.15) is proved at the connecting map between the two rows.

Finally, $E_{3}^{0,5} \simeq \operatorname{Ker}\left(\delta_{2}^{0,5}\right)$ is the kernel $\mathcal{G}$ of the first map appearing in (6.19), and clearly $E_{3}^{0,5} \simeq E_{4,5}^{0,5}$. The map $\delta_{4}^{0,5}$ thus sends this kernel surjectively onto $E_{4}^{4,2} \simeq \Omega_{\pi}(L)^{\oplus a_{2,1}}$, with kernel $E_{5}^{0,5} \simeq E_{\infty}^{0,5}$. The lemma is thus proved.

LEMMA 6.6. In the previous setting, we have

$$
\operatorname{Ext}_{X}^{1}\left(\mathcal{G}, \mathcal{O}_{X}(F-L)\right)=\operatorname{Ext}_{X}^{1}\left(\mathcal{G}, \mathcal{O}_{X}(-F)\right)=0 .
$$




\section{Faenzi, F. Malaspina and G. Sanna}

Proof. We use the exact sequence (6.15). Indeed, let $\mathcal{N}$ be one of the two line bundles $\mathcal{O}_{X}(F-L)$. or $\mathcal{O}_{X}(-F)$, and apply $\operatorname{Hom}_{X}(-, \mathcal{N})$ to $(6.15)$. Set $\mathcal{G}_{i}$ for the image of the $i$ th map $\delta_{2}^{2 i-2,6-i}$ of (6.15). Then our statement is proved if we show that

$$
\operatorname{Ext}_{X}^{i}\left(\operatorname{Ker}\left(\delta_{1}^{2 i-2,6-i}\right), \mathcal{N}\right)=0 \quad \text { for } i=1,2,3 .
$$

Indeed, this would imply $\operatorname{Ext}_{X}^{i+1}\left(\mathcal{G}_{i}, \mathcal{N}\right)=0$ for $i=1,2$, which in turn would give $\operatorname{Ext}_{X}^{1}(\mathcal{G}, \mathcal{N})=0$, which is our statement.

To check (6.20), we look more closely at the defining maps (6.16), (6.17) and (6.18). For $i=1$, we note that (6.16) is constant along the factor $\mathbb{P}^{2}$ of the product $X \simeq \mathbb{P}^{1} \times \mathbb{P}^{2}$, so $\operatorname{ker}\left(\delta_{1}^{0,5}\right)$ is the pull-back to $X$ of a torsion-free sheaf on $\mathbb{P}^{1}$, twisted by $\mathcal{O}_{X}(-L)$. Such a sheaf is then locally free on $\mathbb{P}^{1}$ and therefore splits as a direct sum of line bundles. Actually, the form of (6.16) implies that there are integers $c_{j}$, one for each $j \in \mathbb{N}$ (with only finitely many values of $j \in \mathbb{N}$ satisfying $\left.c_{j} \neq 0\right)$, such that

$$
\operatorname{ker}\left(\delta_{1}^{0,5}\right) \simeq \bigoplus_{j \in \mathbb{N}} \mathcal{O}_{X}(-L-j F)^{\oplus c_{j}}
$$

It follows plainly that $\operatorname{Ext}_{X}^{1}\left(\operatorname{ker}\left(\delta_{1}^{0,5}\right), \mathcal{N}\right)=0$ for our choices of $\mathcal{N}$.

For $i=2$, applying a similar argument to (6.17), we get that there exists a torsion-free sheaf $\mathcal{V}$ on $\mathbb{P}^{2}$ such that

$$
\operatorname{ker}\left(\delta_{1}^{2,4}\right) \simeq \sigma^{*}(\mathcal{V}) \otimes \mathcal{O}_{X}(-F), \quad \mathrm{H}^{0}\left(\mathbb{P}^{2}, \mathcal{V}(-1)\right)=0
$$

Therefore, by Künneth's formula, we have

$$
\operatorname{Ext}_{X}^{2}\left(\operatorname{ker}\left(\delta_{1}^{2,4}\right), \mathcal{O}_{X}(F-L)\right) \simeq \operatorname{Ext}_{\mathbb{P}^{2}}^{2}\left(\mathcal{V}, \mathcal{O}_{\mathbb{P}^{2}}(-1)\right) \otimes \mathrm{H}^{0}\left(\mathbb{P}^{1}, \mathcal{O}_{\mathbb{P}^{1}}(1)\right),
$$

which vanishes because Serre duality and (6.21) imply

$$
\operatorname{Ext}_{\mathbb{P}^{2}}^{2}\left(\mathcal{V}, \mathcal{O}_{\mathbb{P}^{2}}(-1)\right) \simeq \operatorname{Hom}_{\mathbb{P}^{2}}\left(\mathcal{O}_{\mathbb{P}^{2}}, \mathcal{V}(-2)\right)^{\vee}=0 .
$$

The vanishing for $\mathcal{N}=\mathcal{O}_{X}(-F)$ is clear.

For $i=3$, again looking at (6.18), we get a torsion-free sheaf $\mathcal{W}$ on $\mathbb{P}^{2}$ such that

$$
\operatorname{ker}\left(\delta_{1}^{4,3}\right) \simeq \sigma^{*}(\mathcal{W})
$$

This time, Künneth's formula provides $\operatorname{Ext}_{X}^{3}\left(\operatorname{ker}\left(\delta_{1}^{4,3}\right), \mathcal{N}\right)=0$ immediately.

Lemma 6.7. For any sheaf $\mathcal{U}$ which is an extension of copies of $\mathcal{O}_{X}(F-L)$ and $\mathcal{O}_{X}(-F)$, we have $\operatorname{Ext}_{X}^{1}\left(E_{\infty}^{0,5}, \mathcal{U}\right)=0$.

Proof. Clearly, it suffices to check that $\operatorname{Ext}_{X}^{1}\left(E_{\infty}^{0,5}, \mathcal{N}\right)=0$ with $\mathcal{N}=\mathcal{O}_{X}(-F)$ and $\mathcal{N}=\mathcal{O}_{X}(F-$ $L)$. According to Lemma 6.5, we need to check $\operatorname{Ext}_{X}^{1}(\mathcal{G}, \mathcal{N})=0$ and $\operatorname{Ext}_{X}^{2}\left(\Omega_{\pi}(L), \mathcal{N}\right)=0$. The first vanishing comes from Lemma 6.6, and the second one is straightforward.

Now comes the key point. Indeed, the sheaf $\mathcal{F}_{1}$ taken from the filtration $(6.13)$ is an Ulrich sheaf of the form $\mathcal{U}$ as in Lemma 6.7. Therefore, $\mathcal{F}$ is the direct sum of $E_{\infty}^{0,5}$ and $\mathcal{F}_{1}$. But $\mathcal{F}$ is indecomposable; hence, either $\mathcal{F}_{1}=0$ and $\mathcal{F} \simeq E_{\infty}^{0,5}$, or $\mathcal{F} \simeq \mathcal{U}$. In the latter case, Theorem 6.3 is proved, so it remains to analyze the former one. So we assume from now on $\mathcal{F} \simeq E_{\infty}^{0,5}$.

LEMMA 6.8. The sheaf $\mathcal{F} \simeq E_{\infty}^{0,5}$ is isomorphic to $\mathcal{O}_{X}(-L)$ or $\mathcal{O}_{X}(-1)$.

Proof. Since $\mathcal{F} \simeq E_{\infty}^{0,5}$, we have $a_{3,3}=a_{4,4}=0$, so the cohomology table $\left(b_{i, j}\right)$ looks as in the proof of Lemma 6.5. We now argue on whether $\mathrm{H}^{0}(\mathcal{F}(L))$ is zero or not. 


\section{NON-ULRICH REPRESENTATION TYPE}

If $\mathrm{H}^{0}(\mathcal{F}(L)) \neq 0$, looking at the cohomology table of $\mathcal{F}$, we see that $\mathrm{H}^{1}(\mathcal{F}(-L))=\mathrm{H}^{2}(\mathcal{F}(-F-$ $2 L))=0$, and because we are assuming $\mathrm{H}^{0}(\mathcal{F})=0$, we have that item i) of Lemma 6.2 applies to give $\mathcal{F} \simeq \mathcal{O}_{X}(-L)$.

If $\mathrm{H}^{0}(\mathcal{F}(L))=0$, we use once more the vertical Euler sequence, in the form

$$
0 \rightarrow \Omega_{\pi}(-F) \rightarrow \mathcal{O}_{X}(-1)^{\oplus 3} \rightarrow \mathcal{O}_{X}(-F) \rightarrow 0 .
$$

We tensor this sequence with $\mathcal{F}$ and take cohomology. From the cohomology table of $\mathcal{F}$, we extract $\mathrm{H}^{2}\left(\mathcal{F} \otimes \Omega_{\pi}(-F)\right)=0$, which combined with the fact that $\mathcal{F}$ is $\mathrm{ACM}$ gives $\mathrm{H}^{1}(\mathcal{F}(-F))=0$. Also, of course $\mathrm{H}^{2}(\mathcal{F}(-1))=0$, while $\mathrm{H}^{0}(\mathcal{F}(1)) \neq 0$ by assumption. Therefore, item ii) of Lemma 6.2 applies and shows $\mathcal{F} \simeq \mathcal{O}_{X}(-1)$.

This completes the proof of Theorem 6.3.

6.3.1 Proof of Corollaries C and D. The proof of Corollary $\mathrm{C}$ goes as follows. Set $\mathrm{u}(t)=$ $\frac{1}{2}(t+2)(t+1) t$ and note that $\mathrm{u}$ is the reduced Hilbert polynomial of an Ulrich sheaf on $X$ initialized by $t=1$.

Let $\mathcal{F}$ be a semistable ACM bundle on $X$. According to Theorem B, for each indecomposable direct summand $\mathcal{G}$ of the graded bundle $\operatorname{gr}(\mathcal{F})$ provided by a Jordan-Hölder filtration of $\mathcal{F}$, there is some $s \in \mathbb{Z}$ such that $\mathrm{H}^{0}(\mathcal{G}(s))=0$ and $\mathrm{H}^{0}(\mathcal{G}(s+1)) \neq 0$, so $\mathcal{G}(s)$ is one of the sheaves appearing in the next table, where the reduced Hilbert polynomial is also shown:

\begin{tabular}{|c|c|c|c|c|c|}
\hline $\mathcal{G}$ & $\mathcal{O}_{X}(-F)$ & $\mathcal{O}_{X}(F-L)$ & $\Omega_{\pi}(L)$ & $\mathcal{O}_{X}$ & $\mathcal{O}_{X}(-L)$ \\
\hline $\mathrm{p}(\mathcal{G}(s))$ & $\mathrm{u}(t)$ & $\mathrm{u}(t)$ & $\mathrm{u}(t)$ & $\frac{1}{2}(t+2)(t+1)^{2}$ & $\frac{1}{2}(t+1)^{2} t$ \\
\hline
\end{tabular}

Note that these polynomials are pairwise distinct, even upon replacing $t$ with $t+s$ for any $s \in \mathbb{Z}$. Therefore, there is a fixed $s \in \mathbb{Z}$ such that all the summands $\mathcal{G}(s+1)$ are Ulrich bundles (in which case the summands $\mathcal{G}(s)$ are isomorphic to $\mathcal{O}_{X}(-F)$ or $\mathcal{O}_{X}(F-L)$ or $\Omega_{\pi}(L)$ ), or all summands $\mathcal{G}(s)$ are isomorphic to $\mathcal{O}_{X}$, or finally they are isomorphic to $\mathcal{O}_{X}(-L)$. In the last two cases, $\mathcal{G}(s)$ is itself a trivial bundle or a direct sum of copies of $\mathcal{O}_{X}(-L)$. In the first case, the graded bundle $\operatorname{gr}(\mathcal{F}(-s))$ is of the form

$$
\mathcal{O}_{X}(-F)^{\oplus a} \oplus \mathcal{O}_{X}(F-L)^{\oplus b} \oplus \Omega_{\pi}(L)^{\oplus c}
$$

for some integers $(a, b, c)$. Note that there are finitely many ways to choose $a, b, c$ in the above display while keeping the Hilbert polynomial unchanged. This shows that the moduli space of semistable ACM bundles with fixed Hilbert polynomial is a finite set. Corollary $\mathrm{C}$ is proved.

For the proof of Corollary D, we construct the bundles $\mathcal{U}_{k}$ by mutation. Put

$$
\begin{array}{ll}
\mathcal{U}_{-1}=\mathcal{O}_{X}(-F), & \mathcal{U}_{0}=\mathcal{O}_{X}(F-L), \\
\mathcal{U}_{1}=\mathrm{L}_{\mathcal{U}_{0}} \mathcal{U}_{-1}[1], & \mathcal{U}_{k+1}=\mathrm{L}_{\mathcal{U}_{k}} \mathcal{U}_{k-1} \text { for } k \geqslant 1, \\
\mathcal{U}_{-2}=\mathrm{R}_{\mathcal{U}_{-1}} \mathcal{U}_{0}[-1], & \mathcal{U}_{-k-2}=\mathrm{R}_{\mathcal{U}_{-k-1}} \mathcal{U}_{-k} \text { for } k \geqslant 1 .
\end{array}
$$

The fact that the objects $\mathcal{U}_{k}$ are exceptional sheaves having a resolution of the desired form follows as in [FM17, Theorem B].

By Theorem B, any indecomposable rigid ACM bundle on $X$ must be, up to a twist, isomorphic to $\mathcal{O}_{X}(-1), \mathcal{O}_{X}(-L)$ or $\Omega_{\pi}(L)$ or a rigid Ulrich bundle of the form (1.1). In turn, again as in [FM17, Theorem B], a rigid sheaf appearing as the middle term of (1.1) must be isomorphic to $\mathfrak{U}_{k}$ for some $k \in \mathbb{Z}$, with $(a, b)=\left(c_{k-1}, c_{k}\right)$. Moreover, the equality $(a, b)=\left(c_{k-1}, c_{k}\right)$ determines the rigid bundle $\mathfrak{U}_{k}$ uniquely. 


\section{Faenzi, F. Malaspina and G. Sanna}

Finally, given $k \in \mathbb{Z}$, since $\mathcal{U}_{k}^{\vee} \otimes \omega_{X}(2)$ is a rigid Ulrich bundle which fits as middle term of an extension of the form (1.1) with the same values of $a$ and $b$ as $\mathfrak{U}_{1-k}$, by the uniqueness argument for the rigid bundles $\mathcal{U}_{k}$ (cf. again [FM17, Section 2]), we must have $\mathfrak{U}_{1-k} \simeq \mathcal{U}_{k}^{\vee} \otimes \omega_{X}(2)$. This concludes the proof of Corollary D.

\section{ACKNOWLEDGEMENTS}

We are grateful to Gianfranco Casnati for invaluable help. We thank the referees of this paper for the many suggestions allowing a considerable improvement of the paper.

\section{REFERENCES}

Ati57 M.F. Atiyah, Vector bundles over an elliptic curve, Proc. London Math. Soc. 7 (1957), no. 1, 414-452; doi:10.1112/plms/s3-7.1.414.

BBDG06 L. Bodnarchuk, I. Burban, Yu. Drozd and G.-M. Greuel, Vector bundles and torsion free sheaves on degenerations of elliptic curves, in Global Aspects of Complex Geometry (Springer, Berlin, 2006), 83-128; doi:10.1007/3-540-35480-8_\{\}3.

BDG01 I. Burban, Yu. Drozd and G.-M. Greuel, Vector bundles on singular projective curves, Applications of Algebraic Geometry to Coding Theory, Physics and Computation (Eilat, 2001), NATO Sci. Ser. II Math. Phys. Chem., vol. 36 (Kluwer Acad. Publ., Dordrecht, 2001), 1-15; doi:10.1007/978-94-010-1011-5_\{\}1.

BEH87 R.-O. Buchweitz, D. Eisenbud and J. Herzog, Cohen-Macaulay modules on quadrics, Singularities, Representation of Algebras, and Vector Bundles (Lambrecht, 1985), Lecture Notes in Math., vol. 1273 (Springer, Berlin, 1987), 58-116; doi:10.1007/BFb0078838.

BF11 M. C. Brambilla and D. Faenzi, Moduli spaces of rank-2 ACM bundles on prime Fano threefolds, Michigan Math. J. 60 (2011), no. 1, 113-148; doi:10.1307/mmj/1301586307.

BGS87 R.-O. Buchweitz, G.-M. Greuel and F.-O. Schreyer, Cohen-Macaulay modules on hypersurface singularities. II, Invent. Math. 88 (1987), no. 1, 165-182; doi:10.1007/BF01405096.

BM11 E. Ballico and F. Malaspina, Regularity and cohomological splitting conditions for vector bundles on multiprojective spaces, J. Algebra 345 (2011), 137-149; doi:10.1016/j.jalgebra.2011. 08.015 .

CCJ21 O. Calvo-Andrade, M. Corrêa and M. Jardim, Codimension one holomorphic distributions on the projective three-space, Int. Math. Res. Not. 2020 (2020), no. 23, 9011-9074; doi: 10.1093/imrn/rny251.

CFM18 G. Casnati, D. Faenzi and F. Malaspina, Rank two aCM bundles on the del Pezzo fourfold of degree 6 and its general hyperplane section, J. Pure Appl. Algebra 222 (2018), no. 3, 585-609; doi:10.1016/j.jpaa.2017.04.021.

CMP12 L. Costa, R. M. Miró-Roig, and J. Pons-Llopis, The representation type of Segre varieties, Adv. Math. 230 (2012), no. 4-6, 1995-2013; doi:10.1016/j.aim.2012.03.034.

DG01 Yu. A. Drozd and G.-M. Greuel, Tame and wild projective curves and classification of vector bundles, J. Algebra 246 (2001), no. 1, 1-54; doi:10.1006/jabr.2001.8934.

EH87 D. Eisenbud and J. Harris, On varieties of minimal degree (a centennial account), Algebraic Geometry, Bowdoin, 1985 (Brunswick, Maine, 1985), Proc. Sympos. Pure Math., vol. 46 (Amer. Math. Soc., Providence, RI, 1987), 3-13; doi:10.1090/pspum/046.1/927946.

EH88 D. Eisenbud and J. Herzog, The classification of homogeneous Cohen-Macaulay rings of finite representation type, Math. Ann. 280 (1988), no. 2, 347-352; doi:10.1007/BF01456058.

EH92 P. Ellia and A. Hirschowitz, Voie ouest. I. Génération de certains fibrés sur les espaces projectifs et application, J. Algebraic Geom. 1 (1992), no. 4, 531-547. 


\section{NON-ULRICH REPRESENTATION TYPE}

ES09 D. Eisenbud and F.-O. Schreyer, Betti numbers of graded modules and cohomology of vector bundles, J. Amer. Math. Soc. 22 (2009), no. 3, 859-888; doi:10.1090/S0894-0347-08-00620-6.

ESW03 D. Eisenbud, F.-O. Schreyer and J. Weyman, Resultants and Chow forms via exterior syzygies, J. Amer. Math. Soc. 16 (2003), no. 3, 537-579; doi:10.1090/S0894-0347-03-00423-5.

FM17 D. Faenzi and F. Malaspina, Surfaces of minimal degree of tame representation type and mutations of Cohen-Macaulay modules, Adv. Math. 310 (2017), 663-695; doi:10.1016/j.aim. 2017.02 .007$.

FP15 D. Faenzi and J. Pons-Llopis, The CM representation type of projective varieties, 2015, arXiv: 1504.03819.

GK04 A. L. Gorodentsev and S. A. Kuleshov, Helix theory, Mosc. Math. J. 4 (2004), no. 2, 377-440; doi:10.17323/1609-4514-2004-4-2-377-440.

Kac80 V. G. Kac, Infinite root systems, representations of graphs and invariant theory, Invent. Math. 56 (1980), no. 1, 57-92; doi:10.1007/BF01403155.

Mig98 J.C. Migliore, Introduction to liaison theory and deficiency modules, Progr. Math., vol. 165 (Birkhäuser Boston, Inc., Boston, MA, 1998); doi:10.1007/978-1-4612-1794-7.

Mir13 R. M. Miró-Roig, The representation type of rational normal scrolls, Rend. Circ. Mat. Palermo 62 (2013), no. 1, 153-164; doi:10.1007/s12215-013-0113-y.

Ott95 G. Ottaviani, Varietà proiettive di codimensione piccola, Quaderni dell'Indam, vol. 22, Aracne Editrice, 1995.

SE10 F.-O. Schreyer and D. Eisenbud, Betti numbers of syzygies and cohomology of coherent sheaves, ICM (Hyderabad 2010), Proceedings of the ICM 2010, vol. 2 (Hindustan Book Agency, New Delhi, 2010), 586-602.

SS07 D. Simson and A. Skowroński, Elements of the representation theory of associative algebras. Vol. 3. Representation-infinite tilted algebras, London Math. Soc. Stud. Texts, vol. 72 (Cambridge Univ. Press, Cambridge, 2007).

Daniele Faenzi daniele.faenzi@u-bourgogne.fr

Institut de Mathématiques de Bourgogne - UMR CNRS 5584, Université de Bourgogne et Franche-Comté, 9 avenue Alain Savary - BP 47870, 21078 Dijon Cedex, France

Francesco Malaspina francesco.malaspina@polito.it

Dipartimento di Scienze Matematiche, Politecnico di Torino, Corso Duca degli Abruzzi 24, 10129

Torino, Italy

Giangiacomo Sanna giangiacomo.sanna@gmail.com

Institut für Mathematik, Freie Universität Berlin, Arnimallee 3, 14195 Berlin, Germany 